\title{
Effect of proximity of imperfections on buckle interaction in deep subsea pipelines
}

\author{
Hassan Karampour
}

Senior Lecturer, Griffith School of Engineering, Griffith University, Gold Coast Campus, QLD 4222, Australia.E-mail: h.karampour@griffith.edu.au

\begin{abstract}
Lateral buckling of pipelines with closely spaced imperfections laid on even seabed with nonlinear soil-pipe interaction is investigated using finite element analysis (FEA). An analytical solution for lateral buckling of pipeline with single imperfection is proposed and is used to validate the FEA. Effects of amplitude, half wave-length and spacing of imperfections as well as soil resistance on lateral buckling response are presented. It is found that at imperfection spacing greater than half the imperfection half wave-length, there will be no interaction between the buckle lobes. Using validated FEA, the collapse of the pipe-wall due to the interaction between lateral buckling and external pressure is studied. Buckle interaction envelopes are developed and compared to recommendations of the DNV standard. It is shown that in pipeline with closely spaced imperfections, the lateral curvature at the onset of buckling is as large as the critical collapse curvature under combined bending and external pressure.
\end{abstract}


Declarations of interest: none

Keywords:

Lateral buckling

Subsea pipeline

Buckle interaction

Combined bending and external pressure

Cylindrical shell

Imperfection 


\section{Nomenclature}

$a_{i}$

$\mathrm{a}_{\mathrm{o}}$

$\mathrm{D}_{0}$

E

$\mathrm{E}_{\mathrm{t}}$

$\mathrm{F}$

$\mathrm{f}_{0}$

$\mathrm{F}_{\mathrm{y}}$

$\mathrm{f}_{\mathrm{y}}$

$k$

$k_{i}$

$k_{c}$

$k_{L T B}$

$\mathrm{L}_{\mathrm{B}}$

$\mathrm{L}_{\mathrm{G}}$

$\mathrm{L}_{\mathrm{i}}$

L

$\mathrm{M}_{\mathrm{LTB}}$

$\mathrm{M}_{\mathrm{p}}$

$\mathrm{M}_{\mathrm{SD}}$

$\mathrm{N}$

OD

$\mathrm{OD}_{\max }$

$\mathrm{OD}_{\text {min }}$

$\mathrm{P}_{\mathrm{C}}$

$\mathrm{P}_{\mathrm{el}}$

$\mathrm{P}_{\mathrm{p}}$

$\mathrm{P}_{\mathrm{PM}}$

$p_{\mathrm{e}}$

$\mathrm{Q}$

$\mathrm{R}_{\mathrm{i}}$

$r_{i}$

$\mathrm{S}_{\mathrm{SD}}$

$\mathrm{t}$

W

$w_{\mathrm{i}}$

$\bar{W}$

$\alpha_{f a b}$

$\alpha_{c}$

$v$

$\sigma_{\mathrm{y}}$

$\Delta_{\mathrm{i}}$

$\Delta$

$\Omega$

$p_{e}$

$\lambda$

wrinkle amplitude bias

wrinkle imperfection base amplitude

pipe mean diameter, $D_{0}=O D-t$

modulus of elasticity of the pipe

tangent modulus of elasticity of the pipe

axial load in the buckled region

ovalization ratio according to the DNV

yield force $F_{y}=\pi \sigma_{y} D_{0} t$

yield stress in DNV

curvature

lateral imperfection curvature

critical curvature

lateral buckle curvature

Buckle half wave-length

spacing between lateral imperfection lobes

Imperfection half wave-length

length of the shell FE model

moment in the crown of the lateral buckle

plastic moment

design moment

number of half-waves in wrinkle imperfection

nominal outside diameter of pipe

measured maximum outside diameter of pipe

measured minimum outside diameter of pipe

collapse pressure

elastic collapse pressure

buckle propagation pressure

Palmer and Martin propagation pressure

external pressure in DNV

soil resistance in the lateral direction

soil resistance in the axial direction

soil axial resistance parameter

design effective axial force

pipe wall thickness

lateral buckle shape

soil lateral resistance parameter

initial sinusoidal imperfection of the pipeline

fabrication factor in DNV

flow stress parameter in DNV

poison's ratio

yield stress

lateral imperfection amplitude

lateral buckle amplitude

ovalisation ratio

external pressure (used in DNV)

wrinkle imperfection half-wave length 
$\Upsilon \quad$ non-dimensional load

$\gamma_{m}, \gamma_{s c} \quad$ partial resistance factors in DNV

$\bar{\omega} \quad$ localised wrinkle imperfection 


\section{Introduction}

Offshore oil and gas pipelines are subjected to high pressure and high temperature (HP/HT) from the inner hydrocarbon content during operation. Both the rise in temperature and internal pressure may cause longitudinal expansion of the pipeline. This expansion is restrained or semi-restrained by the pipe end devices and the soil which results in build-up of compression stresses in the pipe wall. The large effective axial force may cause formation of global buckle at pipeline locations with high out-of-straightness (OOS). Global buckling may occur in a vertical plane (upheaval buckling) or a horizontal plane (lateral buckling). The former is expected to occur in a buried or trenched pipeline and the latter is common if the pipeline is exposed on the seabed. Uncontrolled lateral buckling is not essentially a failure mode but can cause excessive deformation of the pipeline which could lead to localised buckling collapse, cyclic fatigue failure, fracture or hydrogen induced stress cracking [1]. For instance, the 1.3 million litres oil spill in Guanabara Bay (Brazil, January 2000) was triggered by the increase of pressure and temperature during operation condition which caused a lateral buckle with amplitude of $4 \mathrm{~m}$ and finally resulted in local buckle and rupture of the pipeline [2].

Early studies on global buckling were motivated by lateral buckling of railway tracks [3] using a differential equation approach. Probably Hobbs [4] was the first one to formulate lateral buckling of pipelines. A number of analytical, numerical and experimental studies on lateral buckling of subsea pipelines appeared since then, most notably are [5-12]. The analytical methodology proposed by Hobbs [4] has several limitations due to simplifying assumptions, such as linear elastic material behaviour, Coulomb friction soil model, small rotation theory and imposed post buckling configuration. However, Hobbs' [4] approach is well established in industry and is used as a concept design for susceptibility assessment of global buckling [13]. Hobbs [4] found that in global buckling, the pipeline can deform into four major modes (Fig. 1). Mode 1 requires concentrated lateral forces at each end of the buckle for equilibrium and is 
normally observed in upheaval buckling. In absence of these concentrated forces, the pipeline takes form of mode 3 during lateral buckle. Hobbs [4] noted that mode 3 is associated with highest bending moments and stresses in the pipe, however, asymmetric modes 2 and 4 (Fig. 1) can be triggered at lower axial forces compared to the symmetric mode 3 . Maltby and Calladine [14] presented a simplified solution for lateral buckling of a pipeline with single imperfection on a rigid foundation with nonlinear soil resistance. They assumed bilinear and exponential constitutive models for the lateral soil resistance and found that the condition for localisation of buckling depends mainly on the limiting value of the soil resistance but not much on the exact constitutive law. Miles and Calladine [6] used a small scale experimental rig and observed that during formation of lateral mode 3 in Fig. 1, three distinct lobes develop and gradually approach a point of extinction. They suggested that the physical feature that caused lobes to stop growing was the axial friction that would have to be overcome in the active region of the growing lobes. Karampour et al. [8] conducted analytical and numerical investigations on the lateral buckling of pipelines with single imperfection and found that for a given imperfection amplitude, localisation will take place at a buckle half wave-length at which the axial force is equal to the Euler buckling force. The joint industry projects (JIP), HOTPIPE [15] and SAFEBUCK [16], launched in 2002 and concluded in 2015, established significant knowledge of the design of HP/HT pipelines susceptible to global buckling. The SAFEBUCK [16] study revealed that existing elastic models are excessively conservative and highlighted the necessity of performing FEA with nonlinearity in pipe material and soil properties. Using nonlinear FEA, Brown at al. [17] investigated the wrinkling mode of failure in the pipeline due to excessive bending and Karampour et al. [18-20] studied the interaction between lateral or upheaval buckling and propagation buckling. 
Although lateral buckling of subsea pipelines with isolated imperfections has been extensively reflected upon, the pipeline with closely spaced imperfections has received limited attention [7, 21]. Lateral imperfections due to pipelay can either be intentional (i.e., due to lay route), or unintentional, as-laid variations to the idealised lay route. The unintentional variations to the idealised lay route can have a significant impact on the design for lateral buckling [21] and the interaction between lateral buckling and external pressure [18-20, 22]. In the recent quest for hydrocarbon resources in deeper waters, thick pipes $(15<O D / t<18)$ are exploited due to resilience to propagation buckling [23-28]. Thus, in the current investigation, effect of proximity of imperfections on lateral buckling and buckle interaction of a thick subsea pipeline is studied. In section 2, an analytical solution for lateral buckling of a pipeline with single imperfection is provided. The analytical results are used to validate the FEA which is exploited to investigate the lateral buckling of the pipe with closely spaced imperfections in section 3. Effects of amplitude, half wave-length and spacing of imperfections as well as soil resistance on lateral buckling of the pipeline are investigated. The onset of collapse in the pipewall due to interaction between lateral buckling and external pressure is investigated in section 4 using validated shell FEA and results are compared to DNV [13] recommendations.

\section{Lateral buckling response of a pipeline with single and multiple imperfections}

\subsection{The analytical model}

Tvergaard and Needleman [29] developed an analytical solution for buckling of cylindrical panels under axial compression. Their solution suggested localization of an initially periodic buckling pattern. Subsequent analytical and experimental studies $[5,8]$ confirmed the existence of localized buckle patterns in lateral buckle configuration of pipelines. An analytical solution for lateral buckling of pipeline with single imperfection is developed here based on 
the approach adopted by Tvergaard and Needleman [29]. The pipeline with single imperfection and corresponding soil-pipe interaction model are shown in Figs. $2 \mathrm{a}$ and $2 \mathrm{~b}$ respectively. The pipe is modelled as an elastic beam with length $L_{0}$ resting on an elastic-plastic foundation with softening behaviour which represents the seabed. The pipe has an initial imperfection $\bar{W}(X)$ defined by:

$\bar{W}(X)=\Delta_{0} \sin \left(\pi x / L_{0}\right)$

where $\Delta_{0}$ is the imperfection amplitude. The foundation provides a resisting force per unit length $Q$, with yield point at displacement $W_{0}$ and constitutive law depicted in Fig. $2 \mathrm{~b}$. The softening behaviour of the soil imposes $k_{1}>k_{2}$, where $k_{1}$ is the stiffness before yielding and during unloading and $k_{2}$ is stiffness in the post-yield region. Under an axial load $F$, the pipe deforms laterally to a new equilibrium position shown by $W(X)$, where $W(X)$ is measured from the initial deformed position of the pipe represented by $\bar{W}(X)$ in Fig. 2a. The governing differential equation of the deformed pipe with bending stiffness $(E I)$ is given by:

$E I \frac{d^{4} W}{d X^{4}}+F \cdot \frac{d^{2} W}{d X^{2}}+Q(W)=-F \cdot \frac{d^{2} \bar{W}}{d X^{2}}$

The drag force per unit length $Q$ imposed by the foundation is defined by:

$Q=\left\{\begin{array}{cl}K_{1} W & |W| \leq W_{0} \\ \pm K_{1} W_{0}+K_{2}\left(W \mp W_{0}\right) & |W|>W_{0}\end{array}\right.$

where the upper sign $(+,-)$ operator corresponds to $W>0$ and the lower sign $(-,+)$ operator associates with $W<0$. Introducing non-dimensional parameters of Eq. (4); 
$x=\left(\frac{K_{1}}{E I}\right)^{1 / 4} X \quad \beta=\frac{K_{2}}{K_{1}} \quad w=\frac{W}{W_{0}} \quad \bar{w}=\frac{\bar{W}}{W_{0}} \quad \Upsilon=\frac{1}{2} \frac{F}{\left(K_{1} E I\right)^{1 / 2}}$

and assuming a softening foundation with $\beta<1$, Eq. (2) can be written as;

$$
\begin{array}{ll}
w^{\prime \prime \prime \prime}+2 \Upsilon w^{\prime \prime}+w=-2 \Upsilon \bar{w}^{\prime \prime} & |w| \leq 1 \\
w^{\prime \prime \prime \prime}+2 \Upsilon w^{\prime \prime}+\beta w=-2 \Upsilon \bar{w}^{\prime \prime} \mp(1-\beta) & |w|>1
\end{array} \quad \text { and } \quad()^{\prime}=\frac{d()}{d x}
$$

It's worth noting that for a pipe with imperfection, $\Upsilon<1$ and the upper limit $\Upsilon=1$ corresponds to the Euler buckling load of a straight pipe. The pipeline is considered to be simply supported and thus the displacements and curvatures at either ends are zero; i.e., $w(0)=w\left(L_{0}\right)=0$ and $w^{\prime \prime}(0)=w^{\prime \prime}\left(L_{0}\right)=0$. General solution of differential Eq. (5) is the summation of a homogenous part and a particular part. The homogenous solution yields the deflected shape of a perfectly straight pipe, and the particular solution accounts for the bifurcation from the periodic mode caused by the imposed initial imperfection. The buckling load can be found by solving the linear system of constant coefficients of the form $A \square=B$, where matrices $A$ and $B$ encompass the known parameters, $\beta$ (soil softening parameter), $\Delta_{0}$ (initial imperfection amplitude) and

is a coefficient vector. Coefficient vector $\square$ is found by solving $\Phi=A^{-1} B$ using a symbolic constant coefficient linear system in MATLAB [30]. For sake of brevity the detailed solution procedure is not given here and the avid reader is referred to [22].

\subsection{The Finite Element Model}

A model of a long pipe with a localised sinusoidal imperfection is generated in ANSYS [31]. Due to symmetry only half model in length is created. A single imperfection in the form of Eq. (1) with $L_{0}=100 O D$ is imposed on the pipeline with half- length of $5 L_{0}$. The maximum imperfection amplitude $\Delta_{0}$ is located at the mid-length of the pipe. The pipe is modelled using Beam-188 elements and the element size is taken as half the pipe diameter [8,9]. For 
computational efficiency the lateral frictional drag is approximated using COMBIN39 axially sliding nonlinear spring elements with parameters defined in Fig. 2b. Nonlinear FE analysis is conducted which accounts for the large deformation of the pipe and corresponding softening response of the soil. Longitudinal shrinkage is imposed at the far end of the pipe and the axial force in the buckle region $F_{c r}$ is calculated.

\subsection{Comparison between the analytical and FE results}

Analytical and FE results for a steel pipe (pipe-1) with parameters represented in Table 1 and soil-pipe interaction model of Fig. $2 b$ with $\beta=0.1$, are compared in Fig. 3. The imperfection amplitude $\Delta_{0}$ is increased from $0.1 O D$ to $10 O D$. The buckle forces $F_{c r}$ in Fig. 3 are normalised to the Euler buckling force $F_{e l}=\frac{\pi^{2} E I}{L_{0}^{2}}$ of an ideal column with length of $L_{0}=100 D$.

As shown in Fig. 3 by increasing the imperfection amplitude the buckling forces drop drastically and the results of the FE and analytical solution converge. At $\Delta_{0}>3 O D$, the FE buckling forces are in good agreement with the analytical results.

\section{Lateral Buckling of the pipelines with closely spaced imperfections}

\subsection{The model}

Plan view of the unstressed as-laid configuration of the half-length pipe-1 (Table 1) with two sinusoidal imperfections resting on even seabed is shown in Fig. 4. It should be noted that unlike upheaval buckling response, which is sensitive to the assumed shape of the initial imperfection (because of initial state of stress in the pipe) $[8,31]$, in lateral buckling the pipe 
is initially unstressed and the deformed shape quickly takes one of the Hobbs' mode shapes $[32,33]$. Therefore, assuming imperfections of sinusoidal shape will not affect the accuracy of results. Due to symmetry only half the pipeline in length is modelled. The imperfection lobes 1 and 2 have amplitudes of $\Delta_{1}$ and $\Delta_{2}$ and half wave-lengths of $L_{1}$ and $L_{2}$ respectively and are separated by a distance $L_{G}$. In the FE study that is carried out in this section, the location of lobe 1 is fixed at mid-point $(X=0)$ and the second lobe is shifted along the $X$-axis of the pipeline.

Previous studies $[1,34]$ have shown that a Coulomb friction model is not suitable for design of pipelines with large lateral displacements. Thus, based on the experimental studies of [34], quad-linear lateral and tri-linear axial soil-pipe interaction force-displacement behaviours are adopted. Lateral and axial pipe-soil interaction models are shown in Fig. 5 and the corresponding parameters are presented in Table 2 . The break-out resistances $\left(Q_{1}\right.$ and $\left.S_{0}\right)$ are calculated based on the buoyant weight of pipe-1 in Table 1, with concrete coating thickness of $50 \mathrm{~mm}$ and equivalent Coulomb frictions of 0.5 and 0.57 in axial and lateral directions respectively. The elastic slip in the lateral direction is 2.5 times that of the axial direction $\left(\mathrm{w}_{0}\right.$ $=2.5 \mathrm{r}_{0}$ ) [35]. Previous studies $[8,14]$ have investigated the effect of peak breakout lateral soil resistance $Q_{1}$ on the lateral buckling load of the pipeline. Therefore, effects of the first yield $\left(Q_{0}\right)$ and the residual resistance $\left(Q_{2}\right)$ only are investigated herein.

The pipeline in Fig. 4 is modelled using ANSYS two-node PIPE288 elements [36] with cubic shape function and element size of half the pipe diameter [8,9]. Lateral and axial soil resistances are modelled using sliding unidirectional COMBIN39 spring elements with nonlinear constitutive behaviours shown in Fig. 5 and parameters represented in Table 2. Total length of the pipeline is taken as $L=200 L_{1}$ and localised imperfection lobes 1 and 2 (Fig. 4) are imposed far from the point of application of the axial shortening. The axial shortening $u$ shown in Fig. 
4 is gradually increased at the far end of the pipeline and the non-linear lateral buckling response is captured using the arc-length formulation [37].

\subsection{Critical Buckling Force}

The normalised critical lateral buckling forces of the pipeline with imperfection half-wave lengths of $L_{1}=L_{2}=200 O D$ and $L_{1}=L_{2}=100 O D$ are plotted against normalised separation distances $\left(L_{G} / O D\right)$ in Figs. 6a and $6 \mathrm{~b}$ respectively. The critical loads $\left(F_{c r}\right)$ correspond to the force in the lobe at which the first buckle is triggered and are normalised to the Euler buckling loads $F_{e}^{200}$ and $F_{e}^{100}$ of a straight column with imperfection half-wave lengths of $L_{1}=L_{2}=$ $200 O D$ and $L_{1}=L_{2}=100 O D$ respectively. The solid lines in Fig. 6 correspond to $\Delta_{1}=3.5 O D$ and dashed lines are associated with $\Delta_{1}=7.0 O D$. To investigate the effect of double curvature imperfections on the buckling load, models with $\Delta_{2}=-3.5 O D$ (negative imperfection) were created. The results corresponding to soil parameters with $Q_{0}$ and $Q_{2}$ are referred to as "Normal soil" and are shown with circular markers and those corresponding to soil parameters with $0.5 Q_{0}$ and $0.5 Q_{2}$ are referred to as "Soft soil" and are highlighted with diamond markers in Fig. 6. Normalised curvatures of the initial imperfections are shown in Fig. 6, and the imperfection curvature and the critical curvature are calculated as:

$$
k_{i}=\frac{\pi^{2} \Delta_{i}}{L_{i}^{2}} \quad, \quad k_{c}=\frac{t}{D_{o}^{2}}
$$

The responses show a plateau in the buckle forces at roughly $L_{G}>100 O D$ in Fig. 6a and at $L_{G}>50 O D$ in Fig. 6b. It can be concluded that if the spacing between imperfection lobes is greater than half the initial half-wave length of the imperfection, there will be no interaction 
between the two lobes. In this case the lateral buckling response is similar to response of a pipeline with single isolated imperfection $[6,8]$. The minimum buckle forces occur in pipelines laid on the soft soil with closely spaced imperfection distances $\left(L_{G}=10-20 O D\right)$. There is a difference in the minimum buckle forces of pipeline in Fig. 6a (smaller $k_{i} / k_{c}$ ) and those of pipeline in Fig. $6 \mathrm{~b}\left(\right.$ larger $k_{i} / k_{c}$ ). In the former, the minimum buckle forces are observed at curvatures of the same sign $\left(\Delta_{1} \cdot \Delta_{2}>0\right)$, whereas in the latter the minimum buckle forces are associated with imperfections of the opposite sign $\left(\Delta_{1} \cdot \Delta_{2}<0\right)$. Knowing that $F_{e}^{100}=4 F_{e}^{200}$, it can be inferred from Fig. 6 that the minimum buckling load occurs in the pipeline laid on the soft soil with imperfection curvature of $k_{i} / k_{c}=0.103$. According to Fig. 6 , the buckle forces of pipelines with closely spaced imperfections can be lower than those with single (isolated) imperfections.

Comparison between results of the normal soil and the soft soil in the plateau region of the curves in Fig. 6 suggest that the critical lateral buckling force of the pipeline on the soft soil is in average 10 percent lower than that of the pipe on the normal soil. On the other hand, the reduction in the buckle force by doubling the initial imperfection curvature $\left(k_{i}\right)$ is approximately 57 percent. This suggests that the critical buckle force is predominantly affected by the initial curvature.

\subsection{Post-buckling Response}

The FE results show that the post buckling responses of the pipelines with remotely spaced imperfections $\left(\mathrm{L}_{\mathrm{G}}>100\right)$ are identical. Thus, for the sake of clarity of the presentations, similar results are removed from Figs. 7-9 in this section and only response of $L_{G}=300 O D$ is plotted for comparison. Forces in the buckled region are plotted against normalised lateral buckle 
amplitudes in Fig. 7. The snap-through lateral buckling responses observed in Fig. 7a for pipelines with $k_{i} / k_{c}=0.013$ are similar to the response of pipeline with single imperfection [6, 8]. Peak axial forces are reached at normalised lateral displacements smaller than the breakout displacement of the soil, $\Delta / O D<w_{1}$ (see Table 2). By increasing the imperfection curvatures in Figs. 7c and 7d, the peak axial forces are observed at displacements within the soil yield region $\left(\Delta / O D>w_{2}\right)$. The increase in the axial forces following initial-buckles in Figs. $7 \mathrm{c}$ and $7 \mathrm{~d}$ are due to the initiation and growth of adjacent lateral buckle lobes.

Normalised bending moments in the crown of the pipeline with $L_{1}=L_{2}=100 \mathrm{OD}$ are plotted against normalised buckle amplitudes in Fig. 8 for positive and negative imperfection curvatures. The moments are normalised to the plastic moment [18]:

$$
M_{P}=\sigma_{y} D_{o}^{2} t
$$

As shown in Fig. 8 , in pipelines with $\Delta_{1} \cdot \Delta_{2}>0$, the maximum crown moments $\left(M_{L T B}\right)$ are more augmented in pipes with closely spaced imperfections. In the pipeline with imperfection amplitudes of $\Delta_{1}=7.0 O D$ and $\Delta_{2}=3.5 O D$ shown in Fig. 9c, the plastic bending moments are reached at lateral displacements much smaller than the pipe with isolate single imperfection $\left(\mathrm{L}_{\mathrm{G}}=300 O D\right)$.

Overall, larger bending moments are observed in the pipelines with $\Delta_{1} \cdot \Delta_{2}>0$ compared to pipelines with $\Delta_{1} \cdot \Delta_{2}<0$. This can be explained through comparison of the mode shapes and corresponding lateral curvatures. To do so, lateral buckle configurations of pipeline with $L_{G}=20 O D$, laid on the normal soil with $\Delta_{1}=7 \mathrm{OD}$ and $\Delta_{2}= \pm 3.5 \mathrm{OD}$ are plotted in Fig. 9. Under the same lateral deformation of $\Delta=5 O D$, the crown moment of pipe with $\Delta_{1} \cdot \Delta_{2}>0$ is 60 percent 
higher than that of the pipe with $\Delta_{1} \cdot \Delta_{2}<0$. As shown in Fig.9, both pipes depict Hobbs $3^{\text {rd }}$ mode (Fig.1). However, the adjacent lobe in pipe with $\Delta_{1} \cdot \Delta_{2}<0$ has grown significantly more than the adjacent lobe of the pipe with $\Delta_{1} \cdot \Delta_{2}>0$. The growth in the adjacent lobe relieves the curvature of the first lobe in pipe with $\Delta_{1} \cdot \Delta_{2}<0$. Assuming sinusoidal expressions for the lateral lobes, it is straightforward to show that the lateral buckle curvature $\left(k_{L T B}\right)$ in the crown is equal to:

$$
k_{L T B}=\frac{\pi^{2}}{L_{B}^{2}} \Delta
$$

Using Eq. (8), lateral buckling curvatures $\left(k_{L T B}\right)$ of the pipeline with $\Delta_{1} \cdot \Delta_{2}>0$ is found to be about 55 percent larger than that of the pipe with $\Delta_{1} . \Delta_{2}<0$ which is close to the calculated ratio between crown moments of the pipes (shown in Fig. 9).

Initiation and growth of lateral curvatures in pipelines with different $L_{G}$ are compared in Fig. 10. The initial growth observed in the curvatures is due to the shrinkage of pipe and the post buckling drop in the curvatures are due to the increase in the buckle half-wave length. As shown in Fig. 10, the lateral curvatures in pipes with closely spaced imperfections are substantially larger than the curvature of the pipe with single isolated imperfection $\left(\mathrm{L}_{\mathrm{G}}=300\right)$.

\section{Combined loading}

As shown in section 3, lateral buckling can induce excessive bending moments and curvatures in pipelines with closely spaced imperfections. The combination of large crown moments and curvatures induced by lateral buckling and high external pressure may precipitate catastrophic collapse in the pipe wall. In this section, a FE study is conducted to investigate the 
response of Pipe-1 (Table 1) to pure bending and combined bending and external pressure. First, the FE model is validated against existing experimental study on combined bending and external pressure [38]. Steel pipe-2 in Table 1 with length of $1 \mathrm{~m}(L / O D=31.5)$ was used in the reported experimental study. Using symmetry, a shell FE model of the pipe using half-length $(500 \mathrm{~mm})$ and half cross-section is generated in ANSYS using a total of 2250 SHELL-181 elements (18 elements in circumferential direction and 125 elements along the length). The mesh size was established from a convergence study (not shown here) of the combined loading problem. In the convergence study, the number of elements in the circumferential direction were increased and the corresponding element lengths were selected in a way to generate equilateral elements with small skewness. A bilinear material model (pipe-1 in Table 1) using von-Mises plasticity with isotropic hardening is adopted. To control the numerical solution, a localised wrinkled initial imperfection $\bar{w}[18]$ is imposed on the compression side of the pipe at mid-length as shown in Fig. 10a:

$\bar{w}=-\frac{O D}{2}\left[a_{0}+a_{i} \cos \left(\frac{\pi x}{N \lambda}\right)\right] \cos \left(\frac{\pi x}{N \lambda}\right) \quad 0 \leq x \leq N \lambda$

where the imperfection half-wave length $\lambda=0.165 O D$, number of half-waves $\mathrm{N}=11$, with a base amplitude $a_{0}=0.0025$ and $20 \%$ amplitude bias, $a_{i} / a_{o}$ towards mid-span (Fig. 11a). The load is applied at two stages according to the experiment [38]. At the first stage, a couple is incrementally applied at the far end of the pipe until the desired bending/curvature is achieved. This is followed by incremental application of external hydrostatic pressure while maintaining the desired curvature. Fig. $11 \mathrm{~b}$ shows the normalised moment-curvature response from the experiment and the current FE simulation. 
As seen in Fig. 11b, the bending moment is incremented beyond the plastic moment capacity and held constant at a normalised curvature around 0.55 followed by the application of the external hydrostatic pressure. The onset of failure during the experiment was reported at an applied hydrostatic pressure of $0.3 p_{o}$ accompanied by $20 \%$ drop in moment (Point B in Fig 11b), where $p_{o}$ is given by

$$
p_{0}=2 \sigma_{y}\left(\frac{t}{D_{0}}\right)
$$

The FE results are in good agreement with the experimental results with a predicted collapse at $0.28 p_{o}$ (Point A) accompanied by $14 \%$ drop in moment.

\subsection{External pressure}

To find the collapse pressure of pipe-1 in Table 1, external pressure is imposed on the shell FE model. The collapse mechanism of the pipe under external pressure using experimental and FE methods is comprehensively discussed in [24] and is not repeated here. The collapse pressure of straight pipe-1 in Table 1 with length of $2 \mathrm{~m}(L / O D=8.76)$ and imperfection defined in Eq. 9 is calculated as $P_{c}=77.1 \mathrm{MPa}$ (also indicated in Table. 1). It should be noted that the collapse pressure is independent of the length of the pipe [24].

\subsection{Bending and external pressure}

According to Sec 3.2, the highest bending moment under lateral buckling is in the vicinity of the crown point of the buckle lobe. For this reason, the FE model used to investigate the combined action of bending and external pressure is based on a crown segment of a dented pipe with length $L$. To study the effect of crown length on the combined loading, pipes with 
lengths of $0.6 \mathrm{~m}(L=2.63 O D)$ and $2 \mathrm{~m}(8.76 O D)$ (Fig. 12) are modelled. A half-length and halfsection model is used and the pipe is modelled using SHELL-181 elements with a bi-linear material model using von-Mises plasticity and isotropic hardening. Total of 24 elements with 9 points of integration through the thickness of the shell are used in the circumference of the half-section $(\pi)$ model. The nodes at one end of the model are connected to a master node located at the centre of the pipe, using MPC-184 rigid links. Similar localised wrinkled initial imperfection as described in Sec 4 (Eq. 9 and Fig 11a) is assumed.

Making use of symmetry (Fig 13a), the lateral (X) displacement and the rotation about the longitudinal axis (Z) are restrained along L1 and L2. Similarly, the longitudinal displacement (Z) and rotation about $\mathrm{X}$-axis are restrained along $\mathrm{L} 4$ at mid-length and the vertical $\mathrm{Y}$ displacement is restrained along L3 at the far end. The loading shown in Fig 12 is applied in three steps. In the first step, axial compression load is incremented to the maximum lateral buckling load obtained in Fig 7. In the second load step, the axial load is maintained (which is conservative) while the external hydrostatic pressure is incremented to the desired $P / P_{C}$ ratio of the collapse pressure, $P_{C}$. In the third step of loading, while maintaining the axial load and external pressure achieved in the previous two steps, a couple is incremented at the far end of the pipe until collapse in the pipe wall is initiated (Fig. 13b). It is worth noting that the effect of the axial compression load (step 1) on the final results is negligible. This is expected since the lateral response is dominated by bending (curvature).

Fig. 14 shows the normalised moment-curvature response at different $P / P_{c}$ ratios. For sake of comparison responses with zero external pressure (pure bending) are also shown. As shown in Fig. 14, the ultimate moments under combined bending and pressure occur at lower curvatures in pipe with $L / O D=2.63$. 
Comparison between lateral buckle curvatures in Fig. 10 and critical curvatures in Fig. 14 it can be seen that in pipe with $L_{G}=10 O D$, the maximum lateral curvature is as large as the collapse curvature under bending and external pressure. This is more critical in pipe with $L / O D=2.63$.

\subsection{Combined loading in standards}

In offshore industry, the DNV [39] is widely used. For this reason, the combined action results (bending and external pressure) obtained so far are compared to DNV provisions. According to the DNV, the following criteria shall be fulfilled for local buckling: system collapse (external over pressure only), propagation buckling (external overpressure only) and combined loading (interaction between external or internal pressure, axial load and bending moment). The external pressure $p_{e}$ at any point along the pipeline shall fulfil the following criterion (system collapse check):

$p_{e}-p_{\min } \leq \frac{p_{c}(t)}{\gamma_{m} \gamma_{S C}}$

where $p_{\min }$ is the minimum internal pressure that can be sustained (taken zero for as-laid pipeline), $\gamma_{m}$ is material resistance factor (taken as 1.15 for ultimate limit state) and $\gamma_{S C}$ is safety class resistance factor (taken as 1.138 for medium safety). The characteristic resistance for external collapse pressure $\left(P_{c}\right)$ is calculated from

$\left(P_{c}-P_{e l}\right) \cdot\left(P_{c}^{2}-P_{P}^{2}\right)=P_{c} \cdot P_{e l} \cdot P_{P} \cdot f_{0} \cdot \frac{O D}{t}$

where $f_{0}$ is ovalization (is equal to $0.5 \Omega$ in Eq. 12) and $P_{\mathrm{el}}$ and $P_{\mathrm{P}}$ are given by: 


$$
P_{e l}=\frac{2 E}{\left(1-v^{2}\right)}\left(\frac{t}{O D}\right)^{3} \quad P_{p}=\sigma_{y} \cdot \alpha_{f a b} \cdot \frac{2 \cdot t}{O D}
$$

with $\alpha_{\text {fab }}$ taken equal to one for a seamless pipe. Propagating buckle criterion is given by $(15<O D / t<45)$ :

$$
p_{e}-p_{\text {min }} \leq \frac{p_{p r}}{\gamma_{m} \cdot \gamma_{s c}} \quad p_{p r}=35 \cdot \sigma_{y} \cdot \alpha_{f a b}\left(\frac{t}{O D}\right)^{2.5}
$$

According to the DNV, pipe members subjected to bending moment, effective axial force and external overpressure shall be designed to satisfy the following criterion

$$
\begin{aligned}
& \left\{\gamma_{m} \cdot \gamma_{S C} \cdot \frac{\left|M_{S D}\right|}{\alpha_{c} \cdot M_{P}}+\left\{\frac{\gamma_{m} \cdot \gamma_{S C} \cdot S_{S D}}{\alpha_{c} \cdot S_{P}}\right\}^{2}\right\}^{2}+\left\{\gamma_{m} \cdot \gamma_{S C} \cdot \frac{p_{e}-p_{\min }}{p_{c}}\right\}^{2} \leq 1 \\
& \text { with } \quad \frac{\left|S_{S D}\right|}{S_{P}}<0.4 \text { and } S_{P}=\sigma_{y} \cdot \pi \cdot\left(D_{0}\right) . t
\end{aligned}
$$

which is applicable to pipelines with $15<O D / t<45$. In Eq. (15), $M_{S D}$ is the design moment and $S_{S D}$ is the design effective axial force which is neglected in this work since the response is dominated by bending and external pressure as discussed in [18]. The FE results with different $L / O D$ and DNV recommendations of Eq. (15) are combined into normalised interaction envelopes and are shown in Fig. 15. The DNV recommendation (Eq. 15) does not consider the effect of length (curvature). However, the current results suggest different interaction responses with altered lengths. At $P / P_{c}>0.24$, the short pipe $(L / O D=2.63)$ collapses at lower moments compared to the long pipe $(\mathrm{L} / O D=8.76)$. 


\section{Conclusions}

Finite element investigations of lateral buckling of deep subsea pipelines with closely spaced imperfections were provided. The results showed that if the spacing between imperfection lobes is greater than half the initial imperfection half-wave lengths, there will be no interaction between the two lobes and the lateral buckling response will be similar to a pipeline with single imperfection. The critical buckling forces were found to be predominantly governed by the imperfection curvatures rather than the lateral soil resistance. Maximum lateral moments were observed in pipelines with closely spaced imperfections of the same sign $\left(\Delta_{1} \cdot \Delta_{2}>0\right)$. It was shown that in the pipeline with $L_{G}=10 O D$ and $\Delta_{1}=+2 \Delta_{2}$, the crown moment reaches the plastic moment at lateral amplitudes as small as 5OD. Collapse of the pipe under combined bending and external pressure was investigated by modelling the crown segment of the lateral buckle with different lengths. The length effect is neglected in the interaction expression recommended in DNV. However, current results showed that the collapse of the pipe under combined bending (due to lateral buckling) and external pressure is affected by the length of the crown segment of the pipe. Comparison between lateral buckling curvatures and critical curvatures under combined loading showed that in pipe with $L_{G}=10 O D$, the lateral curvature at the onset of buckling is as large as the collapse curvature under bending and external pressure.

\section{References}

[1] Bai Y, Bai Q. Subsea pipelines and risers: Elsevier; 2005. 
[2] da Costa AM, dos Santos Amaral C, de Oliveira Cardoso C, Andueza A. Soil-structure interaction of heated pipeline buried in soft clay. 2002 4th International Pipeline Conference: American Society of Mechanical Engineers; 2002. p. 457-66.

[3] Kerr AD. Analysis of thermal track buckling in the lateral plane. Acta Mechanica. 1978;30:17-50.

[4] Hobbs RE. In-service buckling of heated pipelines. Journal of Transportation Engineering. 1984;110:175-89.

[5] Croll JG. A simplified model of upheaval thermal buckling of subsea pipelines. ThinWalled Structures. 1997;29:59-78.

[6] Miles D, Calladine C. Lateral thermal buckling of pipelines on the sea bed.

TRANSACTIONS-AMERICAN SOCIETY OF MECHANICAL ENGINEERS JOURNAL OF APPLIED MECHANICS. 1999;66:891-7.

[7] Taylor N, Gan AB. Submarine pipeline buckling - imperfection studies. Thin-Walled Structures. 1986;4:295-323.

[8] Karampour H, Albermani F, Gross J. On lateral and upheaval buckling of subsea pipelines. Engineering Structures. 2013;52:317-30.

[9] Zeng X, Duan M. Mode localization in lateral buckling of partially embedded submarine pipelines. International Journal of Solids and Structures. 2014;51:1991-9.

[10] Zhu J, Attard MM, Kellermann DC. In-plane nonlinear localised lateral buckling of straight pipelines. Engineering Structures. 2015;103:37-52.

[11] Li G, Zhan L, Li H. An analytical solution to lateral buckling control of subsea pipelines by distributed buoyancy sections. Thin-Walled Structures. 2016;107:221-30.

[12] Neto AG, Mattos Pimenta Pd, Martins CdA. Hydrostatic pressure load in pipes modeled using beam finite elements: theoretical discussions and applications. Journal of Engineering Mechanics. 2017;143:04017003.

[13] Veritas DN. DNV-RP-F110. Global Buckling of Submarine Pipelines-Structural Design to High Temperature/High Pressure. 2007.

[14] Maltby TC, Calladine CR. An investigation into upheaval buckling of buried pipelinesII. Theory and analysis of experimental observations. International journal of mechanical sciences. 1995;37:965-83.

[15] Collberg L, Mork K, Levold E, Vitali L. Hotpipe JIP: design guidelines for HP/HT pipelines. OMAE2005-67523, Proceedings of the 24h International Conference on Offshore Mechanics and Arctic Engineering, Halkidiki, Greece2005.

[16] Bruton D, Carr M, Crawford M, Poiate E. The safe design of hot on-bottom pipelines with lateral buckling using the design guideline developed by the safebuck joint industry project. Proceedings of the Deep Offshore Technology Conference, Vitoria, Espirito Santo, Brazil2005.

[17] Brown G, Brunner M, Qi X, Stanley I. Lateral buckling reliability calculation methodology accounting for buckle interaction. Offshore Technology Conference: Offshore Technology Conference; 2006.

[18] Karampour H, Albermani F, Veidt M. Buckle interaction in deep subsea pipelines. ThinWalled Structures. 2013;72:113-20.

[19] Karampour H, Albermani F. Buckle interaction in textured deep subsea pipelines. Ships and Offshore Structures. 2016;11:625-35.

[20] Karampour H, Albermani F. Experimental and numerical investigations of buckle interaction in subsea pipelines. Engineering Structures. 2014;66:81-8.

[21] Igland RT, Kvittem MI, Vysochinskiy D. Expansion Design Philosophy to Prevent Buckle Walking at Very Uneven Seabed. ASME 2009 28th International Conference on Ocean, Offshore and Arctic Engineering: American Society of Mechanical Engineers; 2009. p. 243-8. 
[22] Karampour H, Albermani F, Major P. Interaction Between Lateral Buckling and Propagation Buckling in Textured Deep Subsea Pipelines. ASME 2015 34th International Conference on Ocean, Offshore and Arctic Engineering: American Society of Mechanical Engineers; 2015. p. V003T02A79-VT02A79.

[23] Stephan P, Love C, Albermani F, Karampour H. Experimental study on confined buckle propagation. Advanced Steel Construction. 2016;12:10.

[24] Albermani F, Khalilpasha H, Karampour H. Propagation buckling in deep sub-sea pipelines. Engineering Structures. 2011;33:2547-53.

[25] Yu J-x, Sun Z-z, Liu X-X, Zhai Y-x. Ring-truss theory on offshore pipelines buckle propagation. Thin-Walled Structures. 2014;85:313-23.

[26] Xue J, Wang Y, Yuan D. A shear deformation theory for bending and buckling of undersea sandwich pipes. Composite Structures. 2015;132:633-43.

[27] Karampour H, Alrsai M, Albermani F, Guan H, Jeng D-S. Propagation Buckling in Subsea Pipe-in-Pipe Systems. Journal of Engineering Mechanics. 2017;143:04017113.

[28] Alrsai M, Karampour H, Albermani F. Numerical study and parametric analysis of the propagation buckling behaviour of subsea pipe-in-pipe systems. Thin-Walled Structures. 2018;125:119-28.

[29] Tvergaard V, Needleman A. On localized thermal track buckling. International Journal of Mechanical Sciences. 1981;23:577-87.

[30] MathWorks I. MATLAB : the language of technical computing : computation, visualization, programming : installation guide for UNIX version 5: Natwick : Math Works Inc., 1996.; 1996.

[31] Maltby TC, Calladine CR. An investigation into upheaval buckling of buried pipelines-II. Theory and analysis of experimental observations. International Journal of Mechanical Sciences. 1995;37:965-83.

[32] Wang Z, Tang Y, van der Heijden GHM. Analytical study of distributed buoyancy sections to control lateral thermal buckling of subsea pipelines. Marine Structures. 2018;58:199-222.

[33] Miles DJ, Calladine CR. Lateral Thermal Buckling of Pipelines on the Sea Bed. Journal of Applied Mechanics. 1999;66:891-7.

[34] White D, Cheuk C. Modelling the soil resistance on seabed pipelines during large cycles of lateral movement. Marine Structures. 2008;21:59-79.

[35] Bruton D, White D, Cheuk C, Bolton M, Carr M. Pipe/soil interaction behavior during lateral buckling, including large-amplitude cyclic displacement tests by the safebuck JIP. Offshore Technology Conference: Offshore Technology Conference; 2006.

[36] Tanizawa KM, Koryo Stress Analysis of a Concave Polyhedral Shell University of Tokyo; 1973.

[37] Crisfield M. An arc-length method including line searches and accelerations.

International journal for numerical methods in engineering. 1983;19:1269-89.

[38] Corona E, Kyriakides S. On the collapse of inelastic tubes under combined bending and pressure. International Journal of Solids and Structures. 1988;24:505-35.

[39] Veritas DN. Offshore standard dnv-os-f101. Submarine pipeline systems. 2017. 

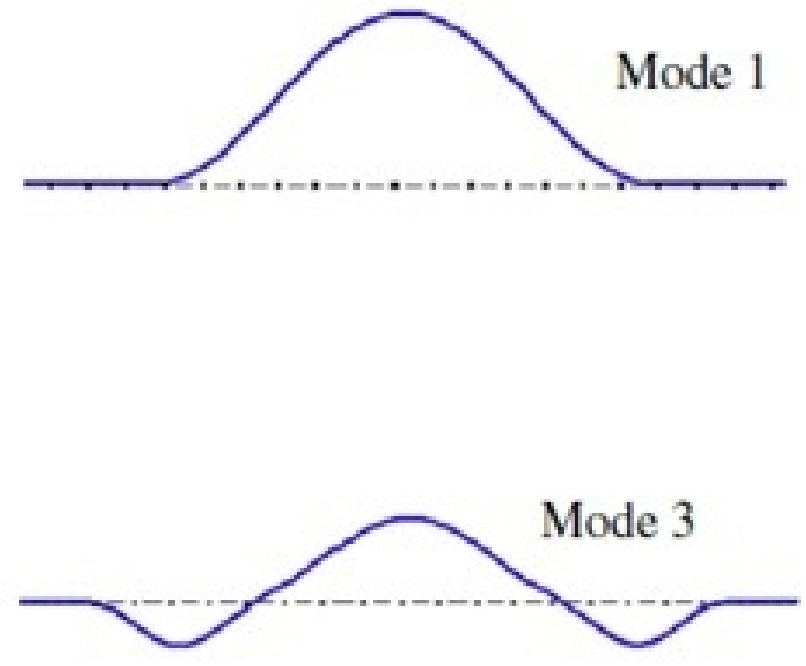

Mode 2

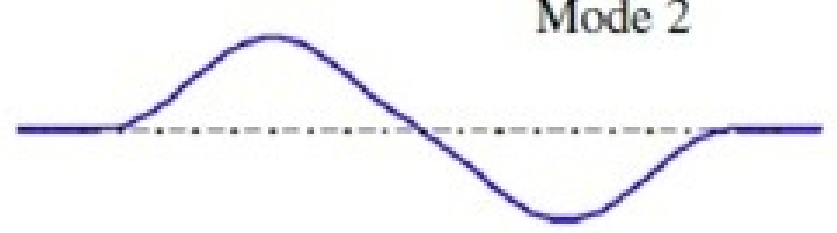

Mode 4

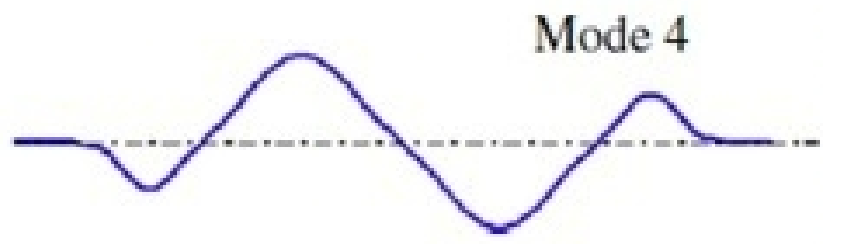




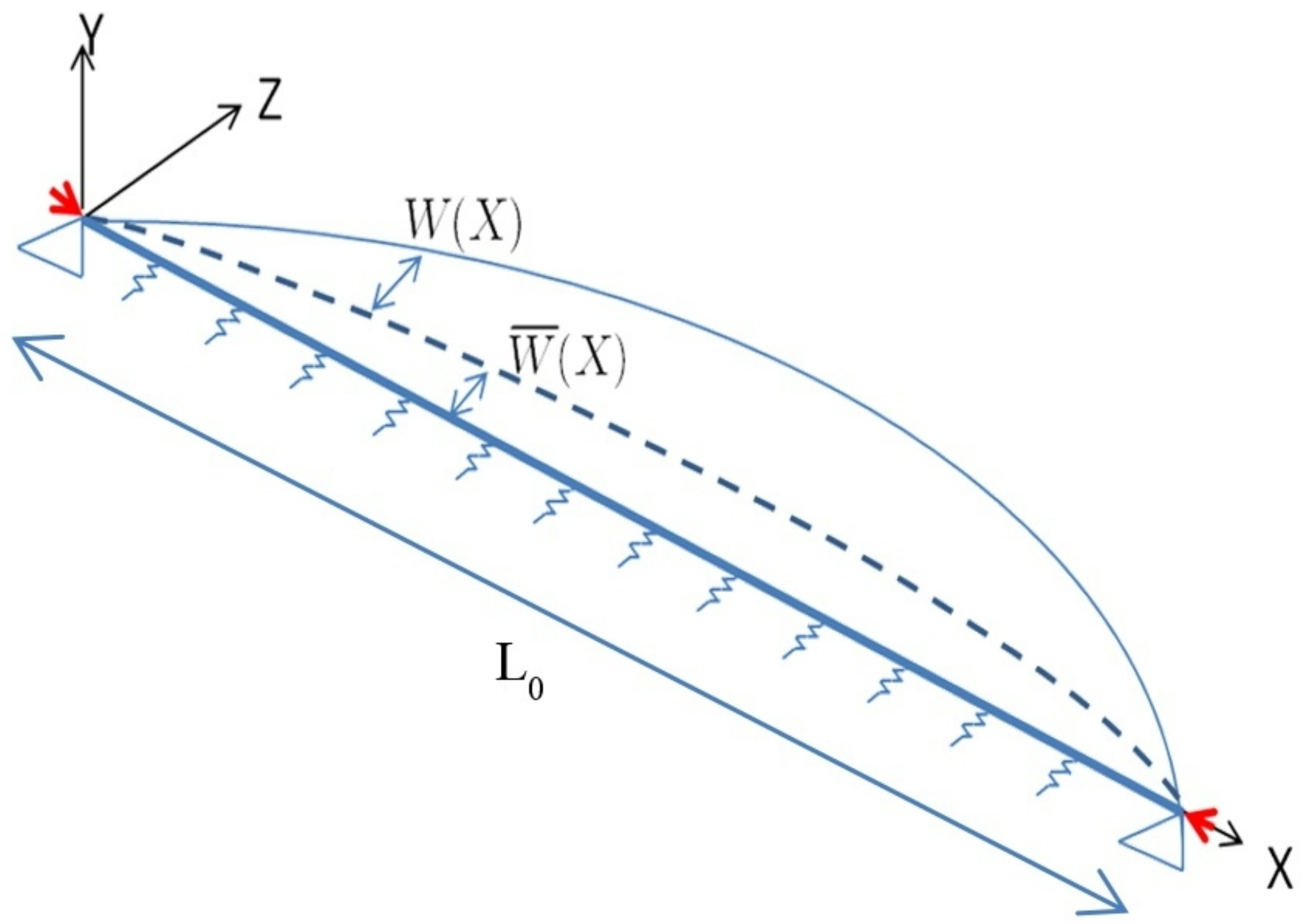




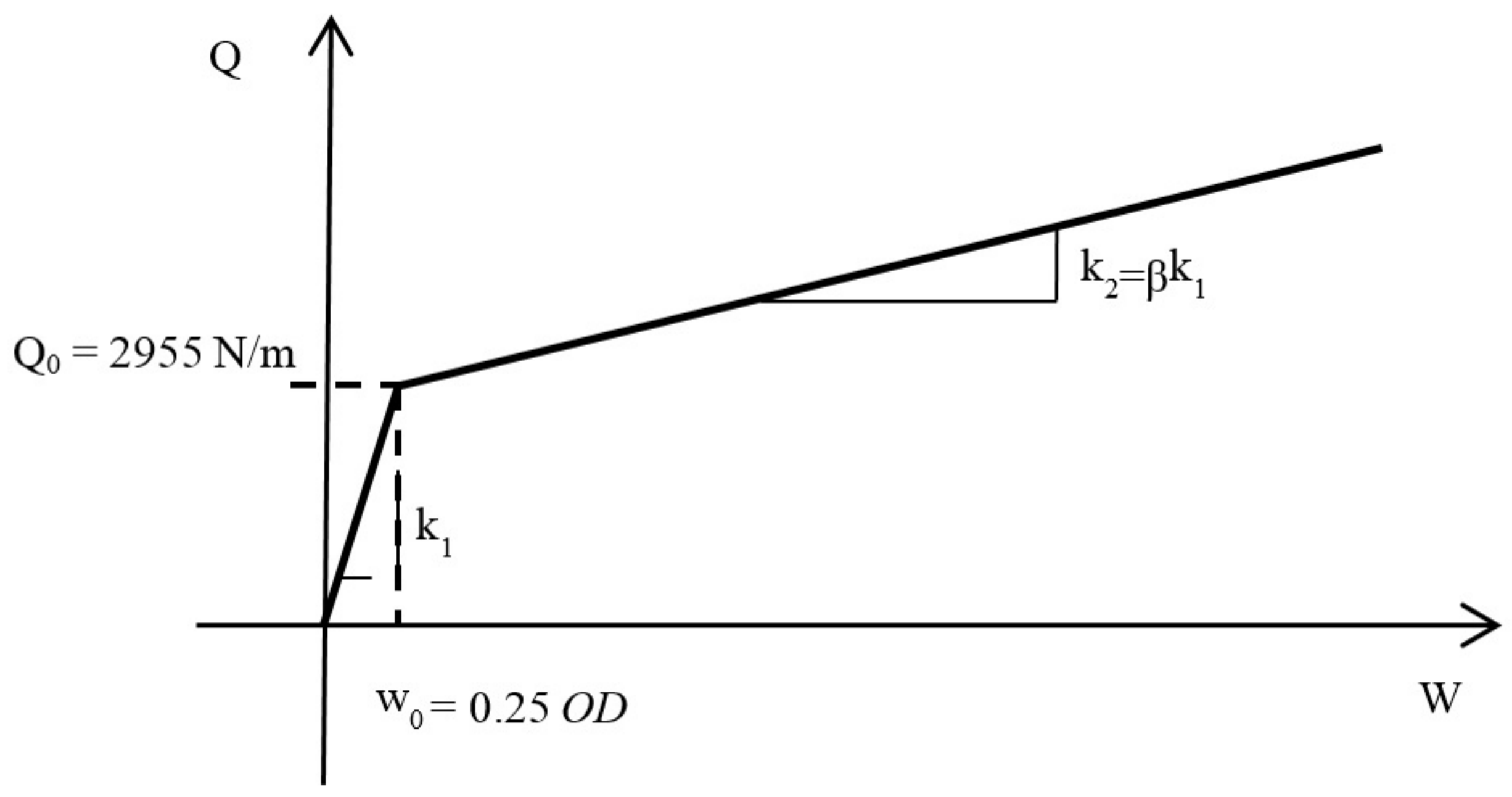




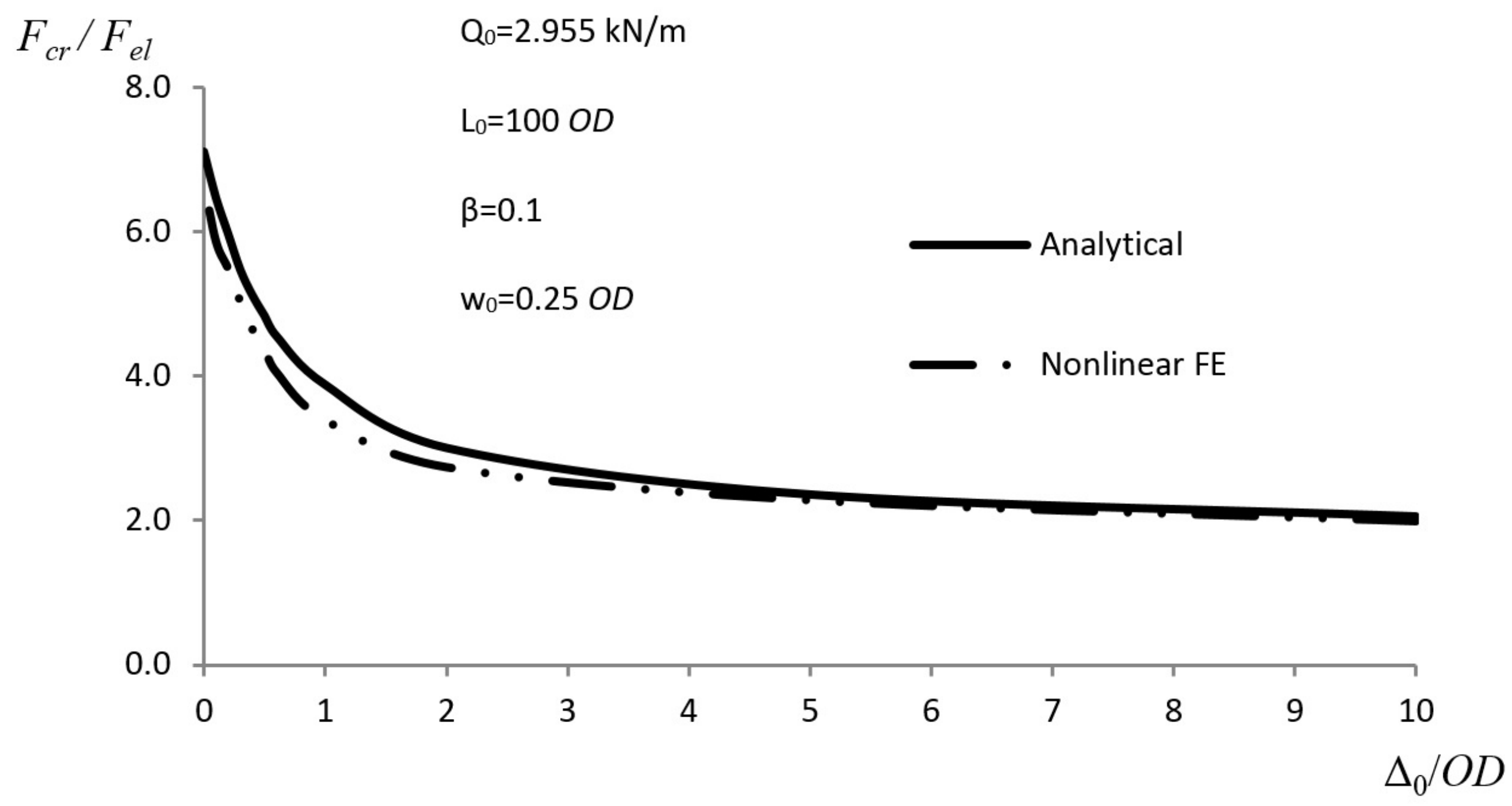




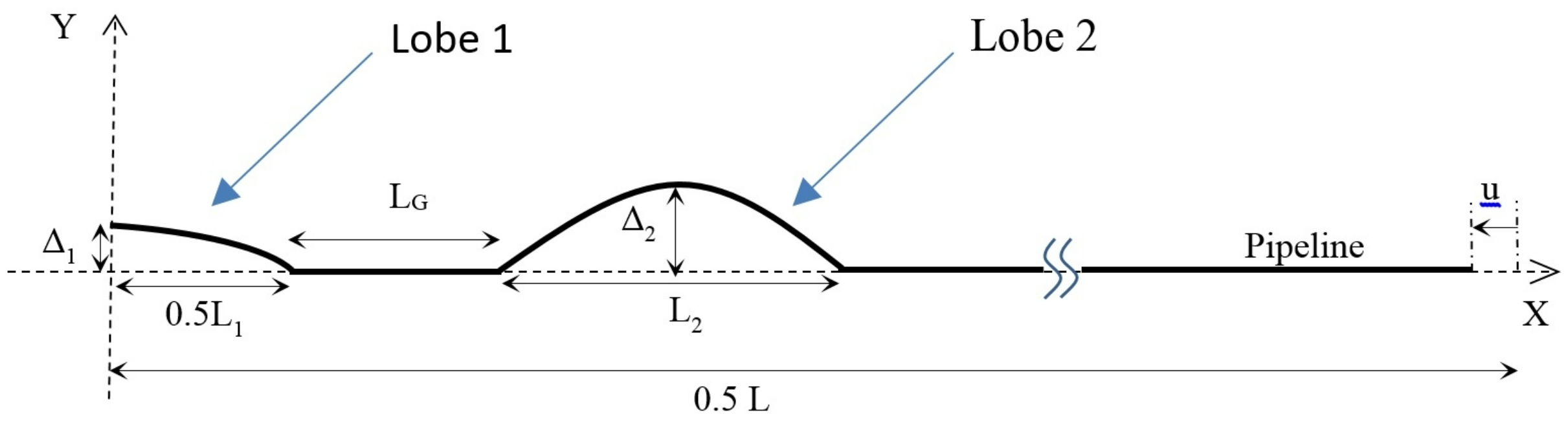




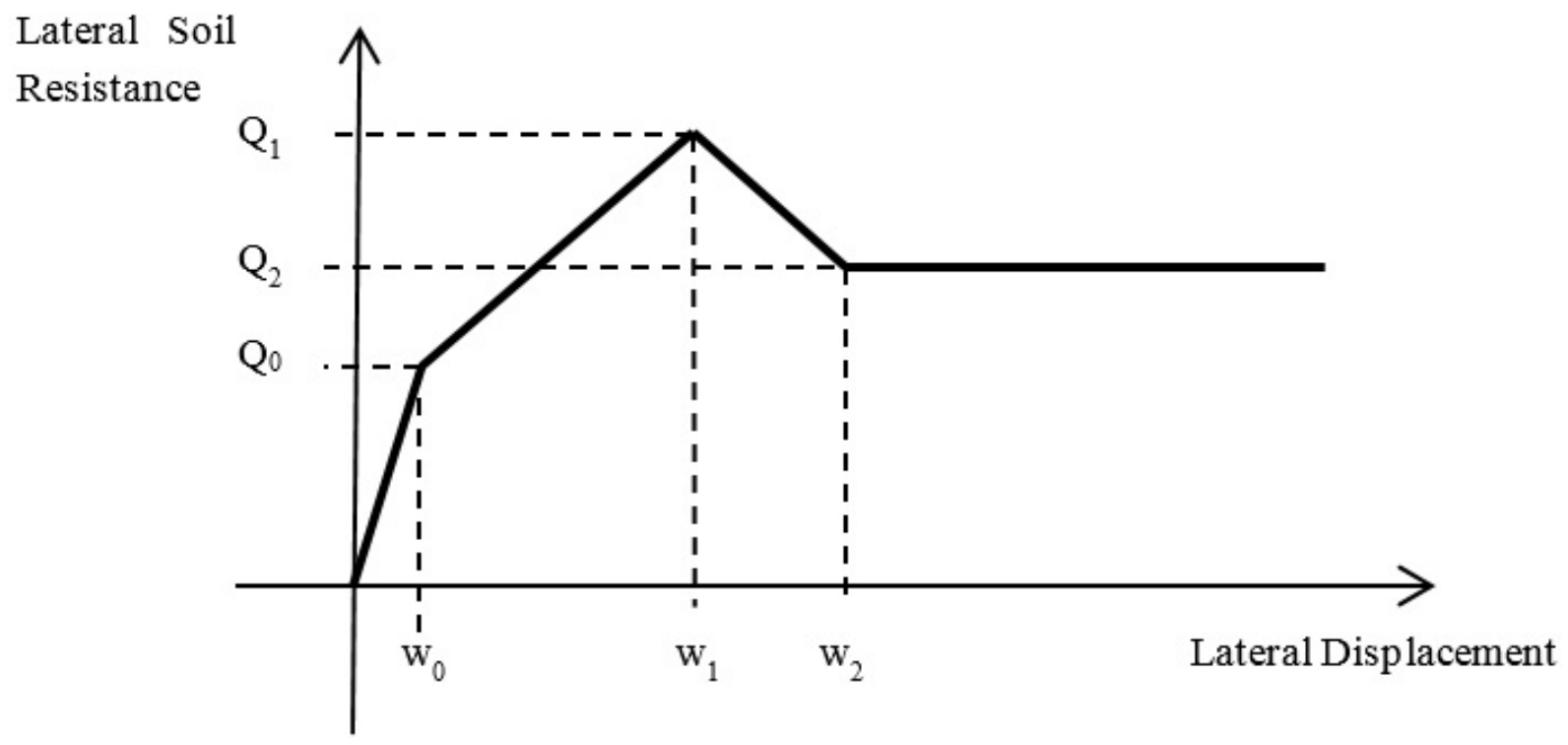

Lateral soil resistance

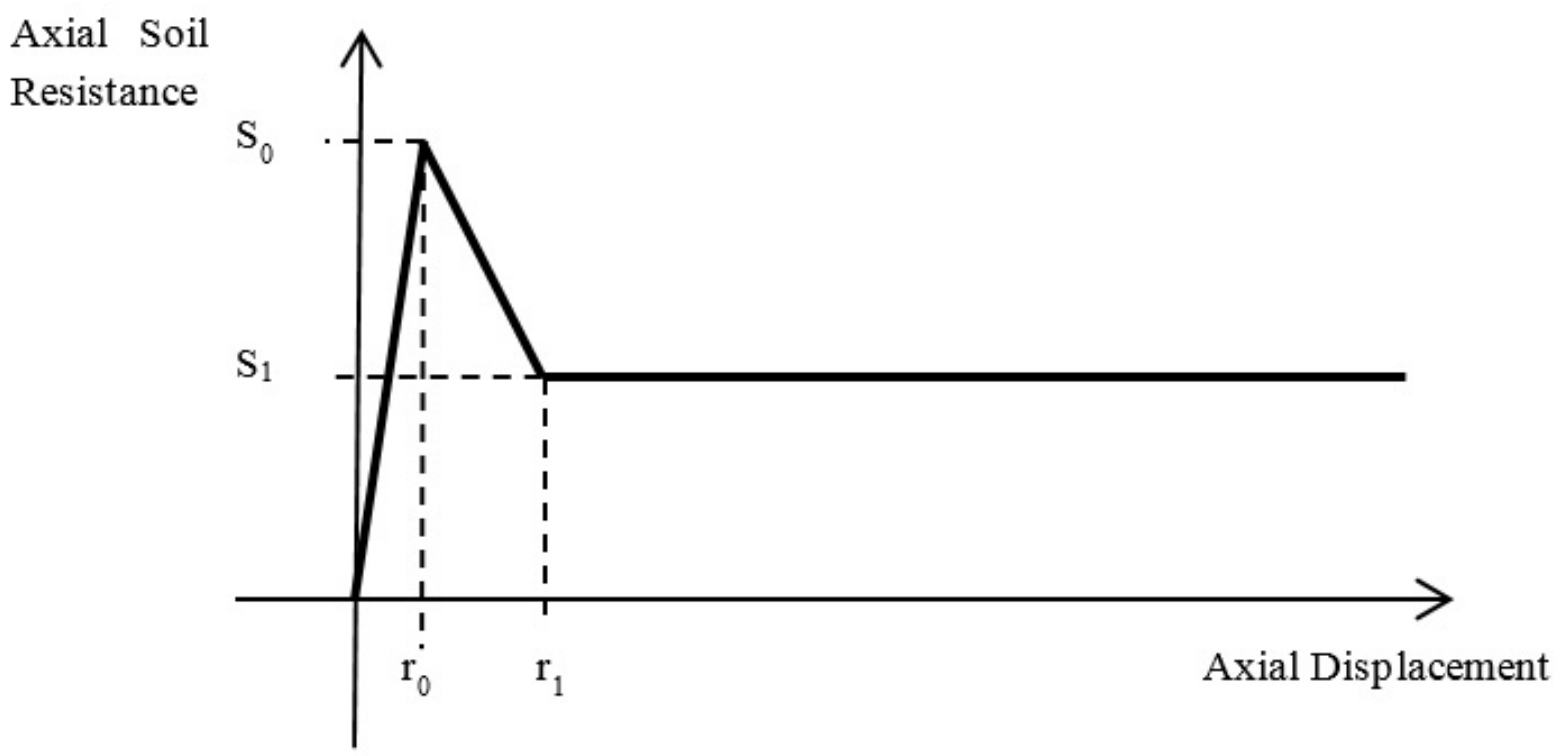

Axial soil resistance 


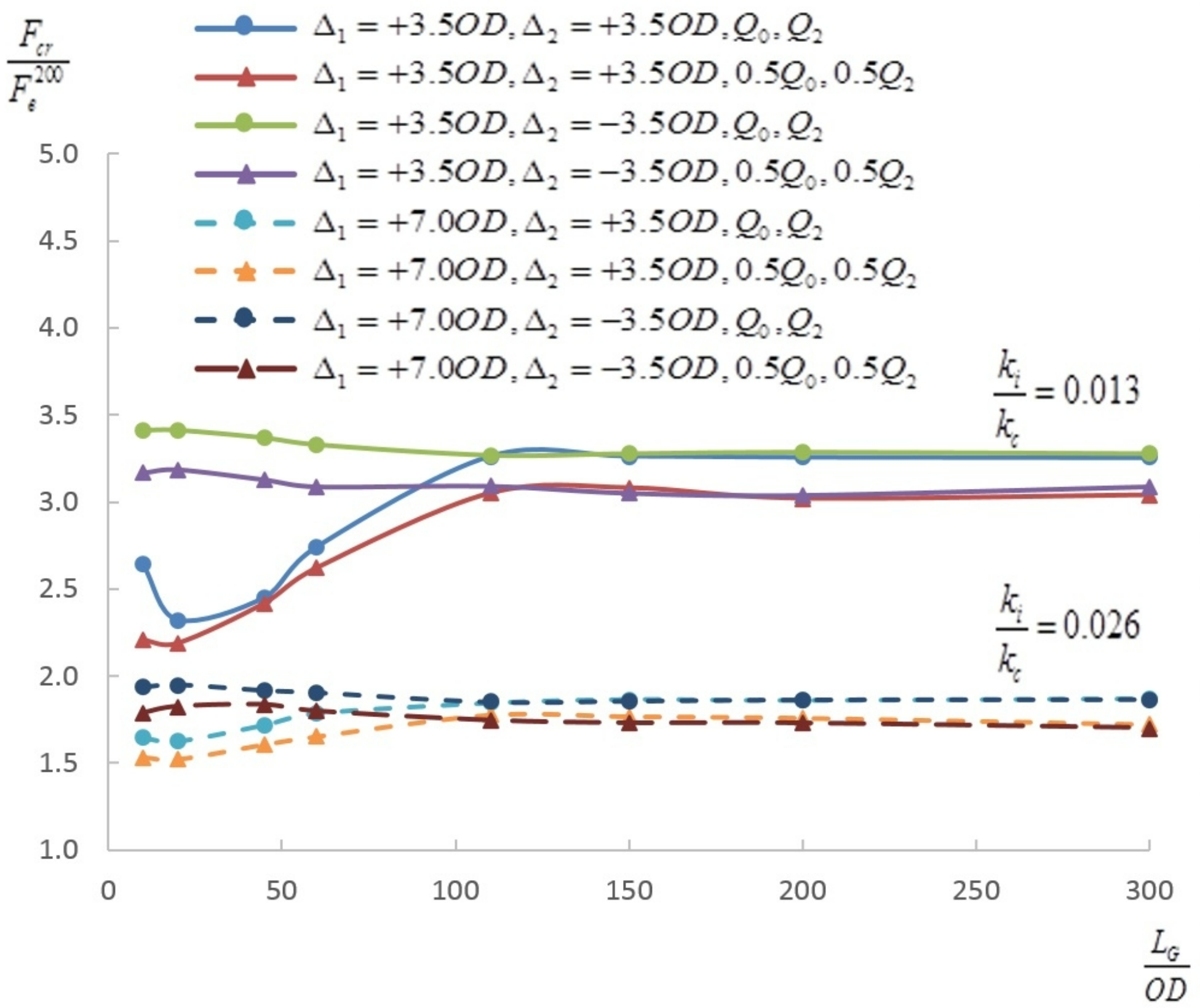




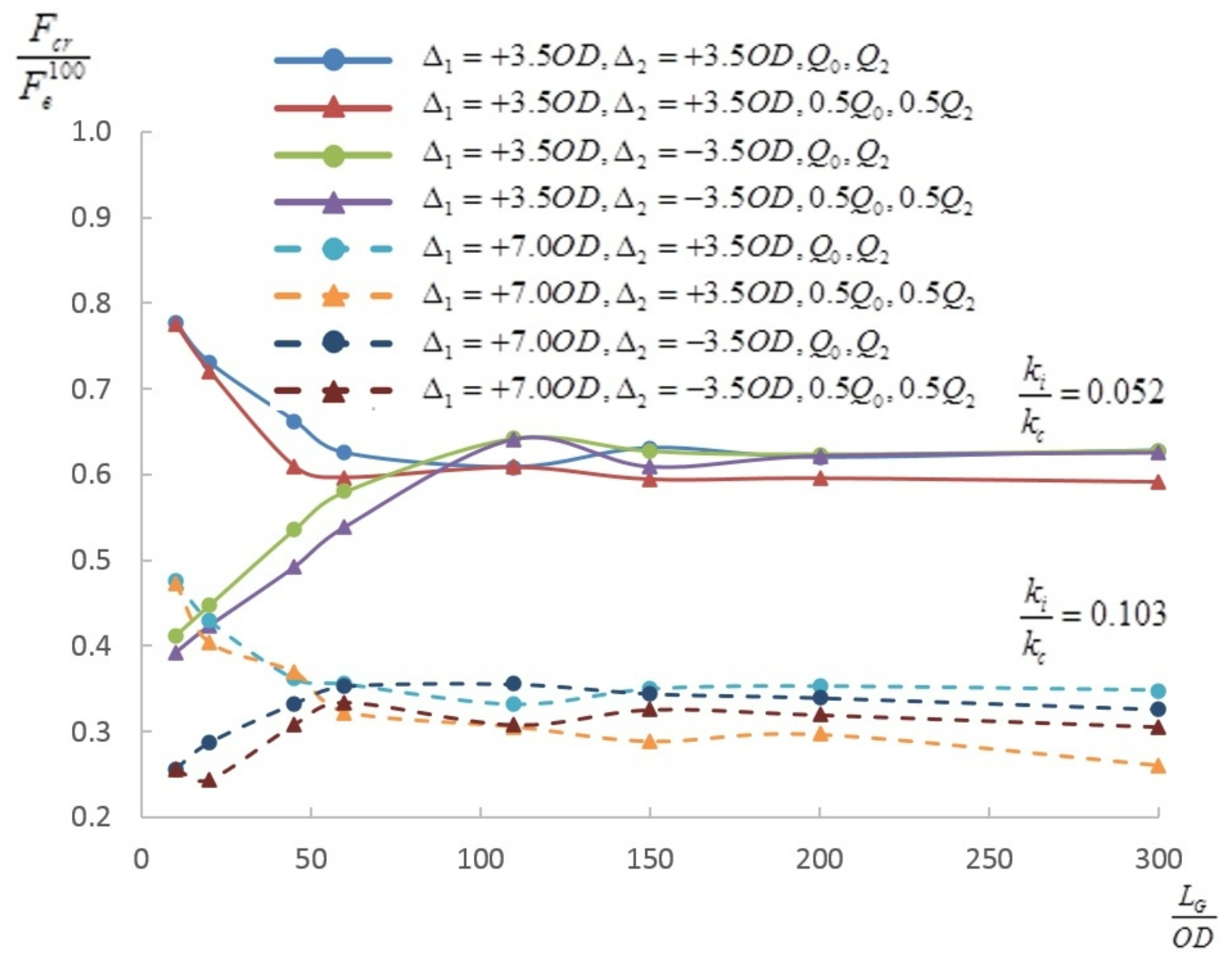



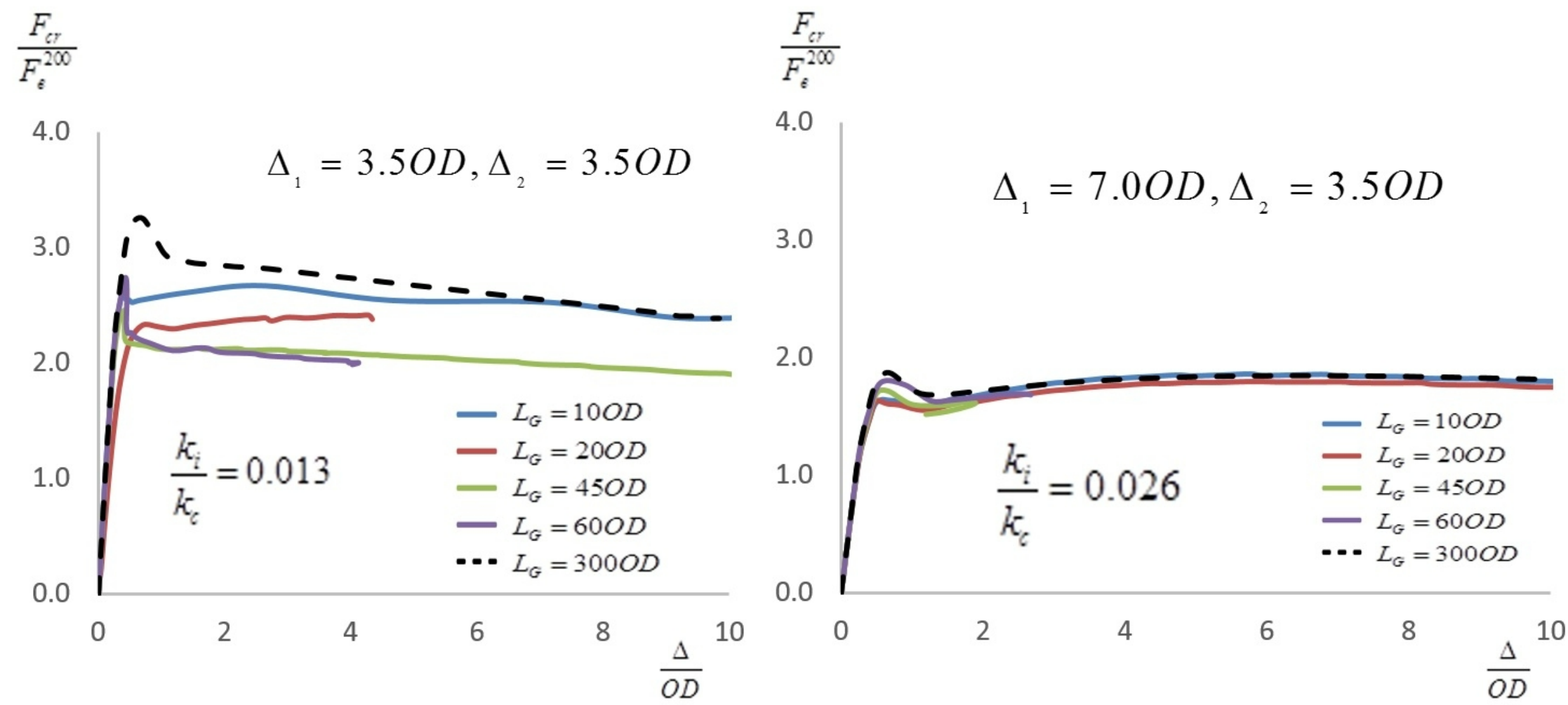

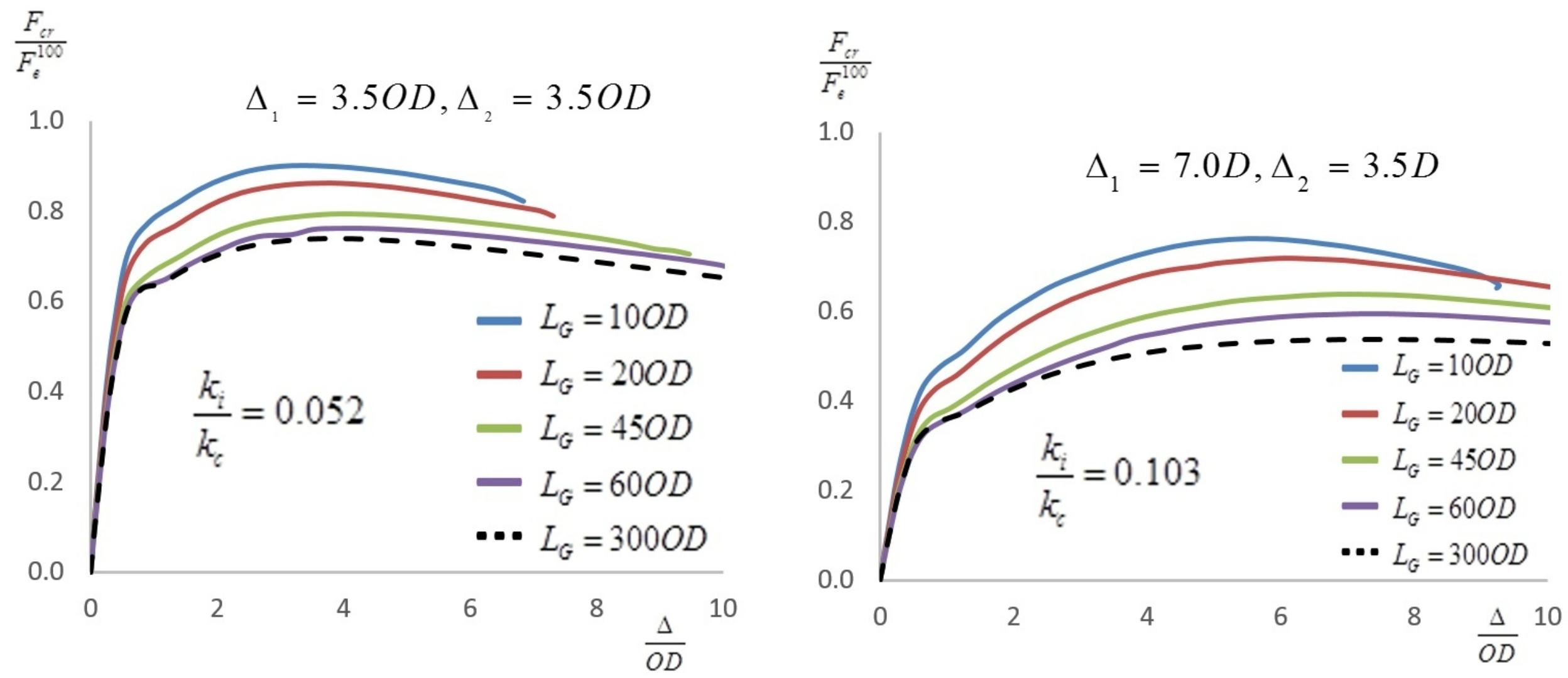

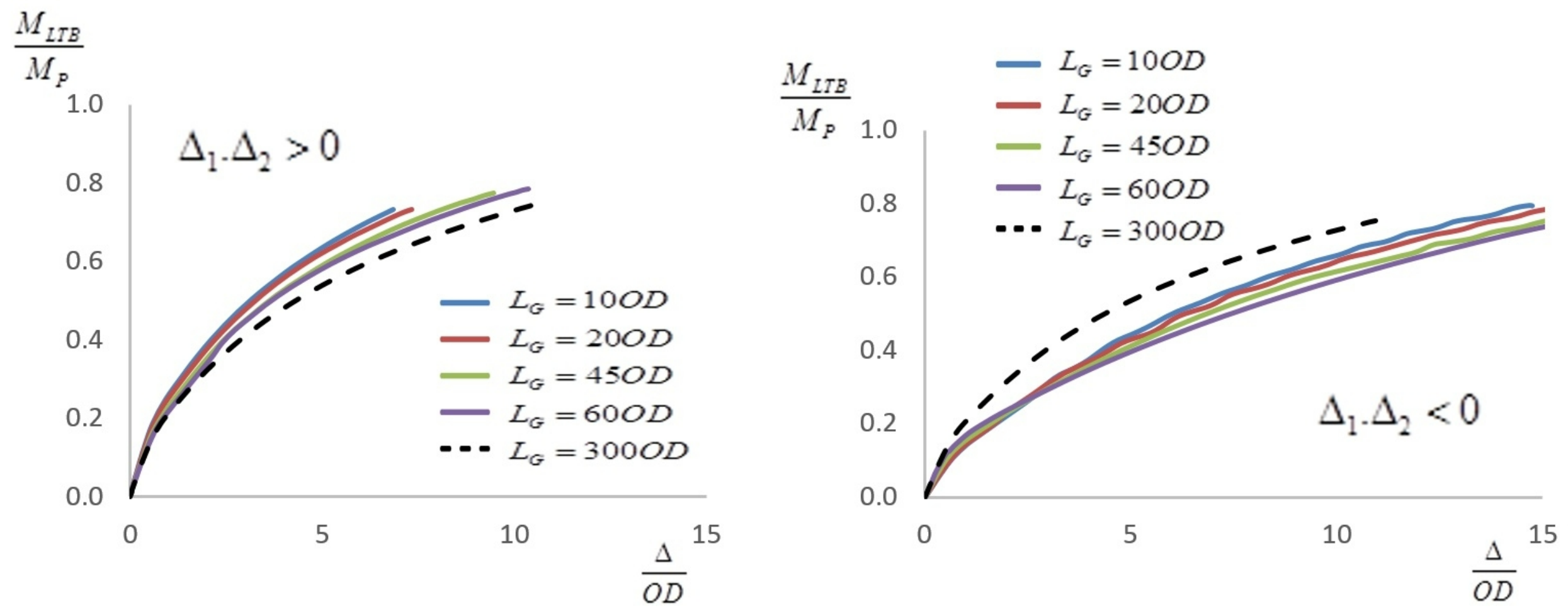


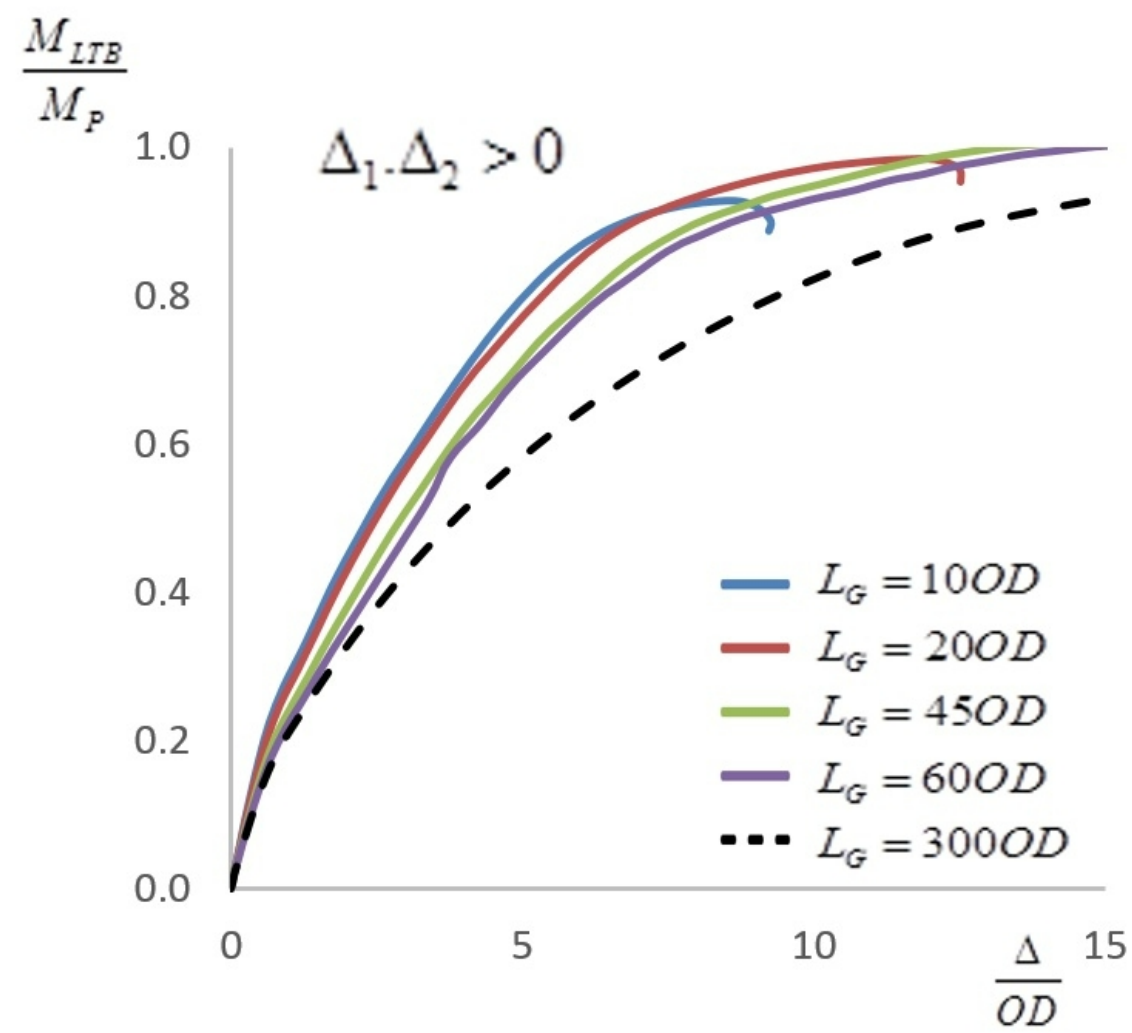

$\frac{M_{\text {LTB }}}{M_{P}}$

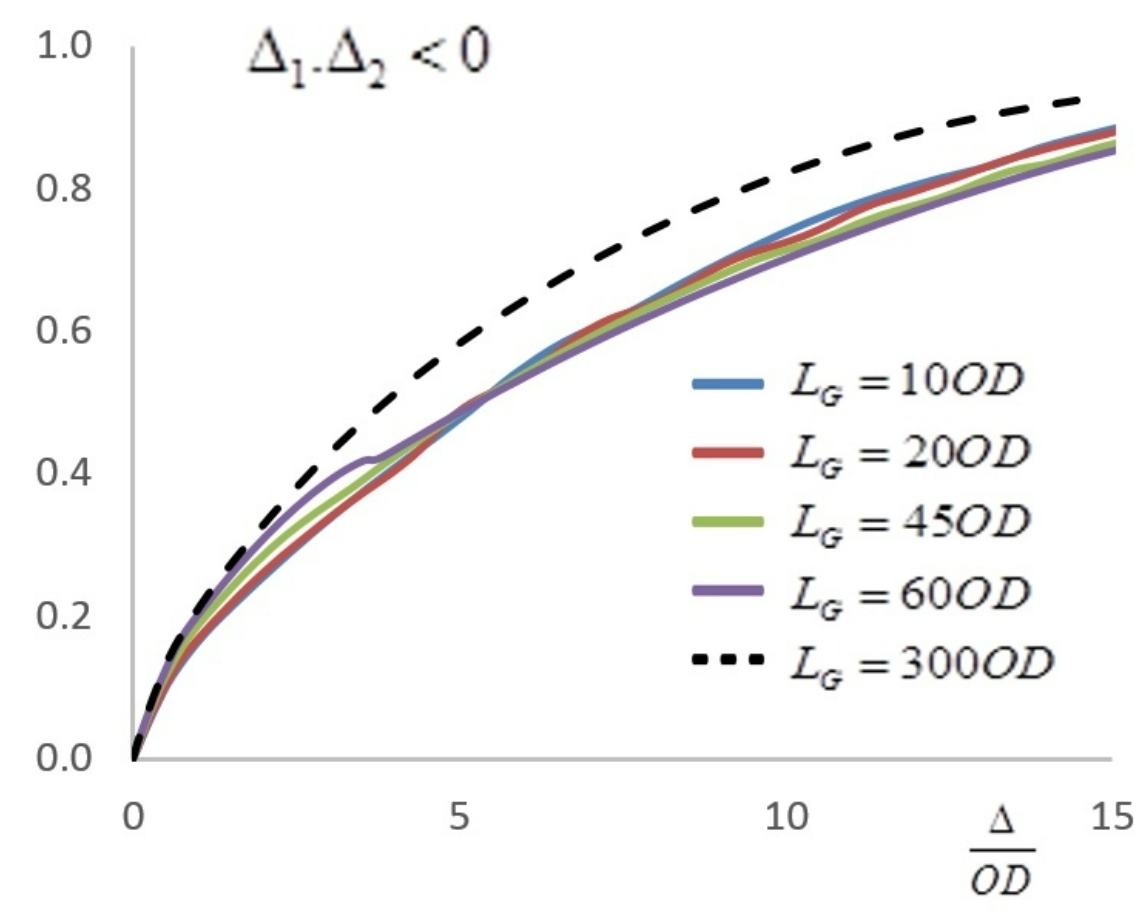




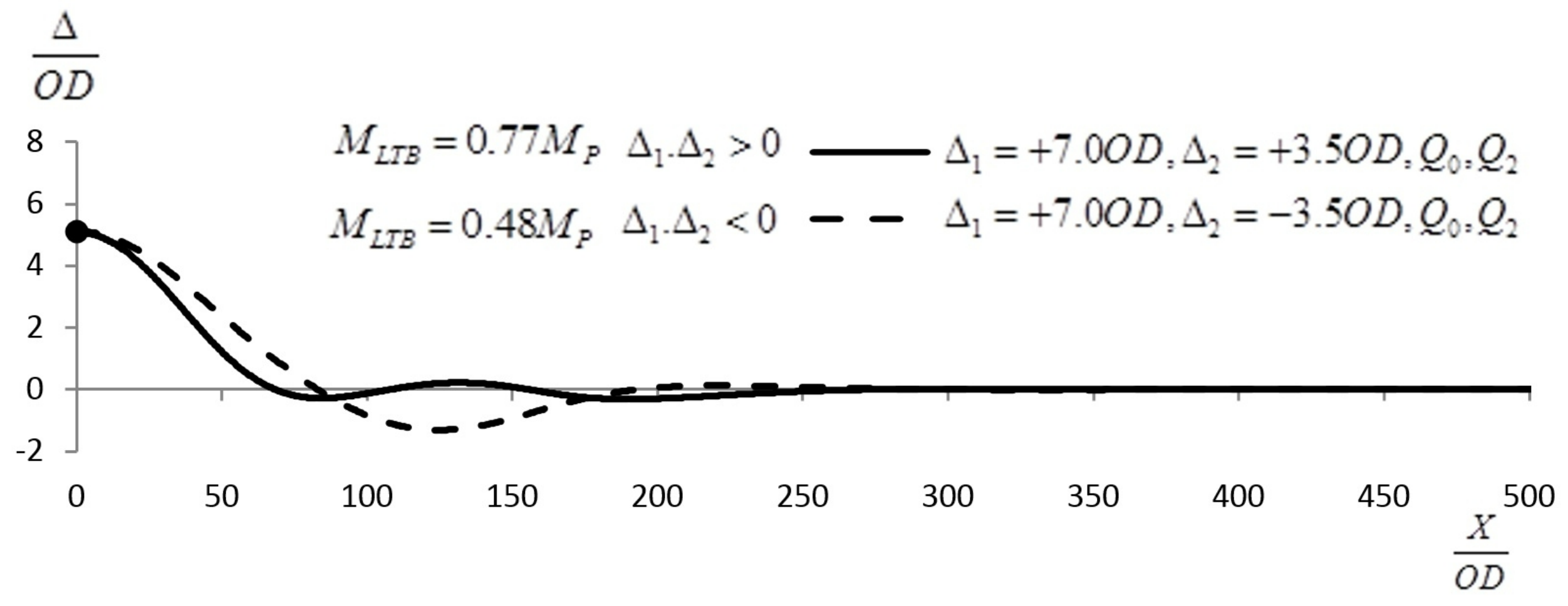




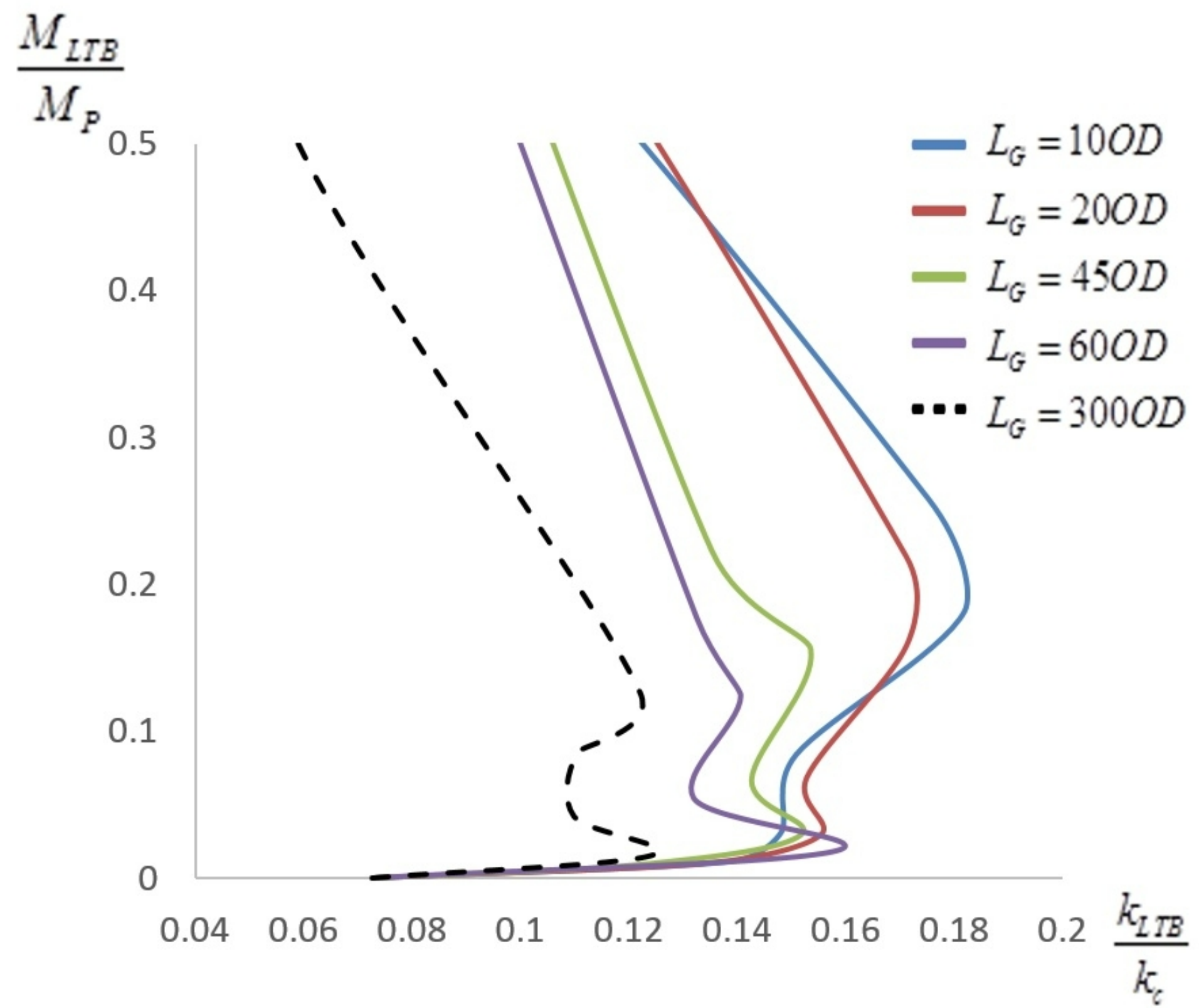



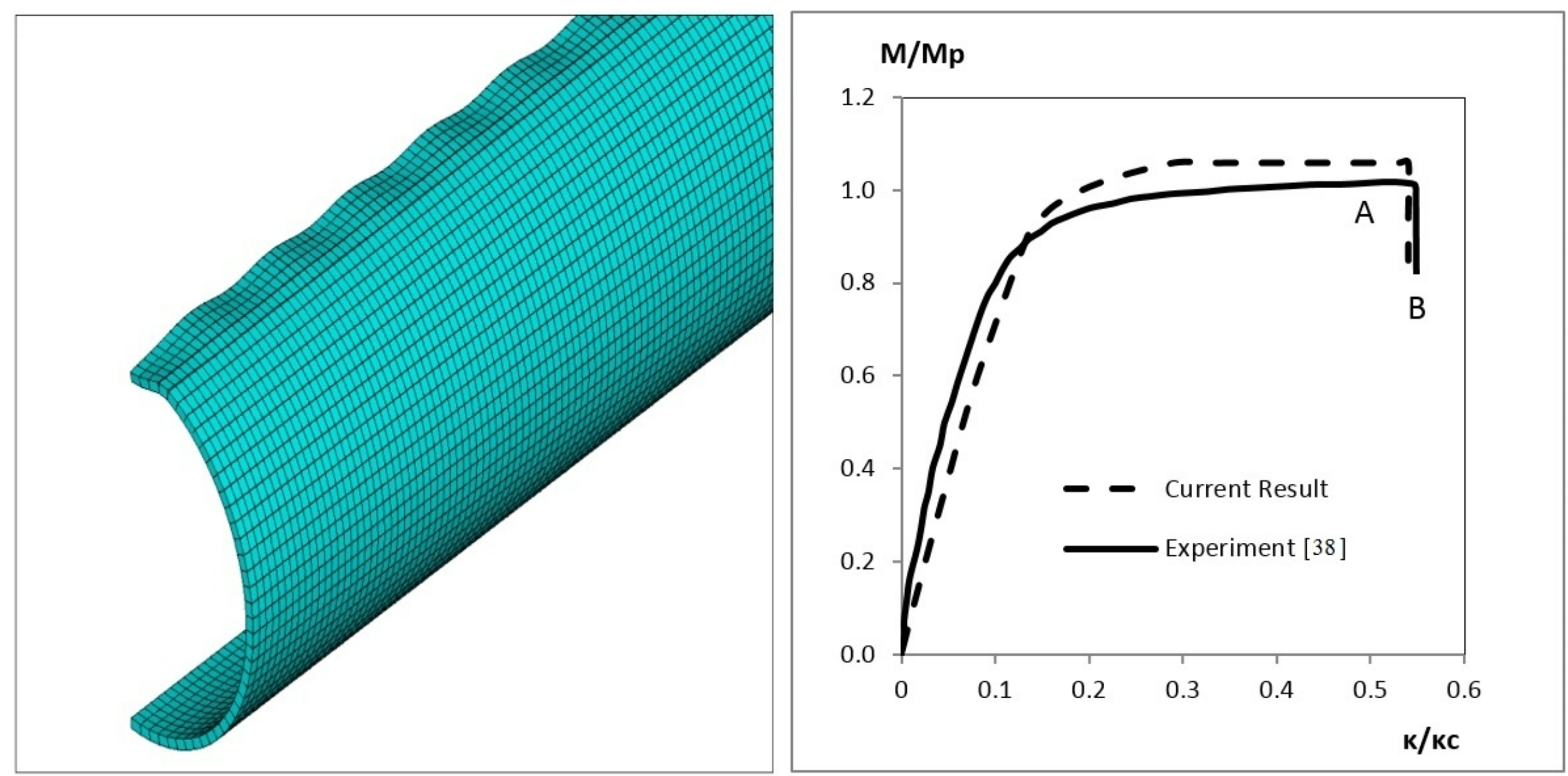


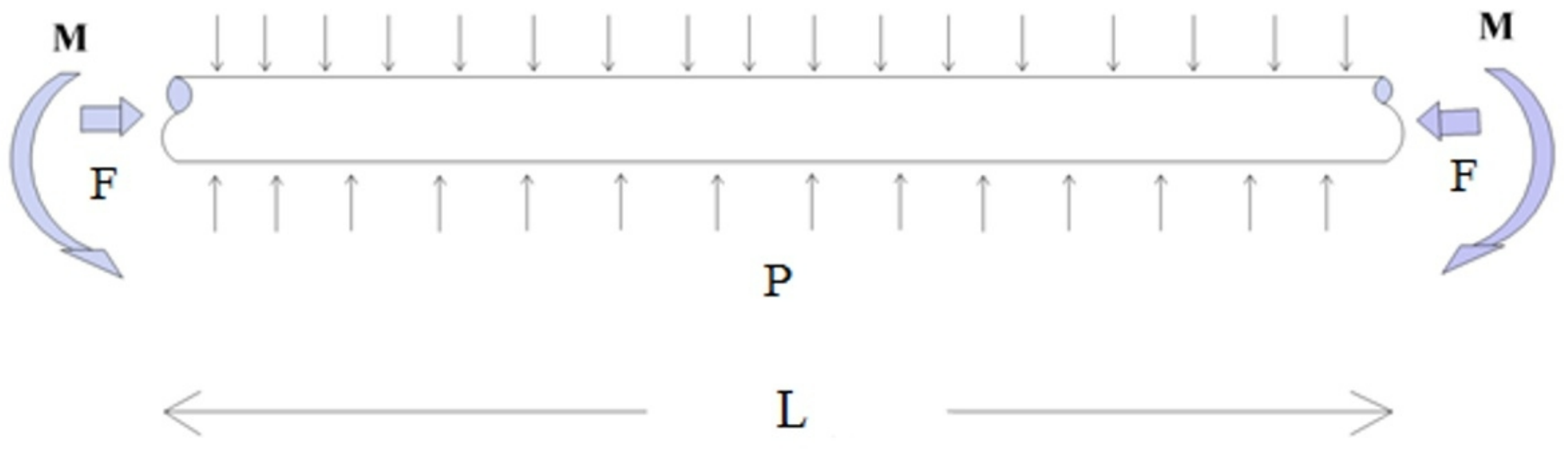



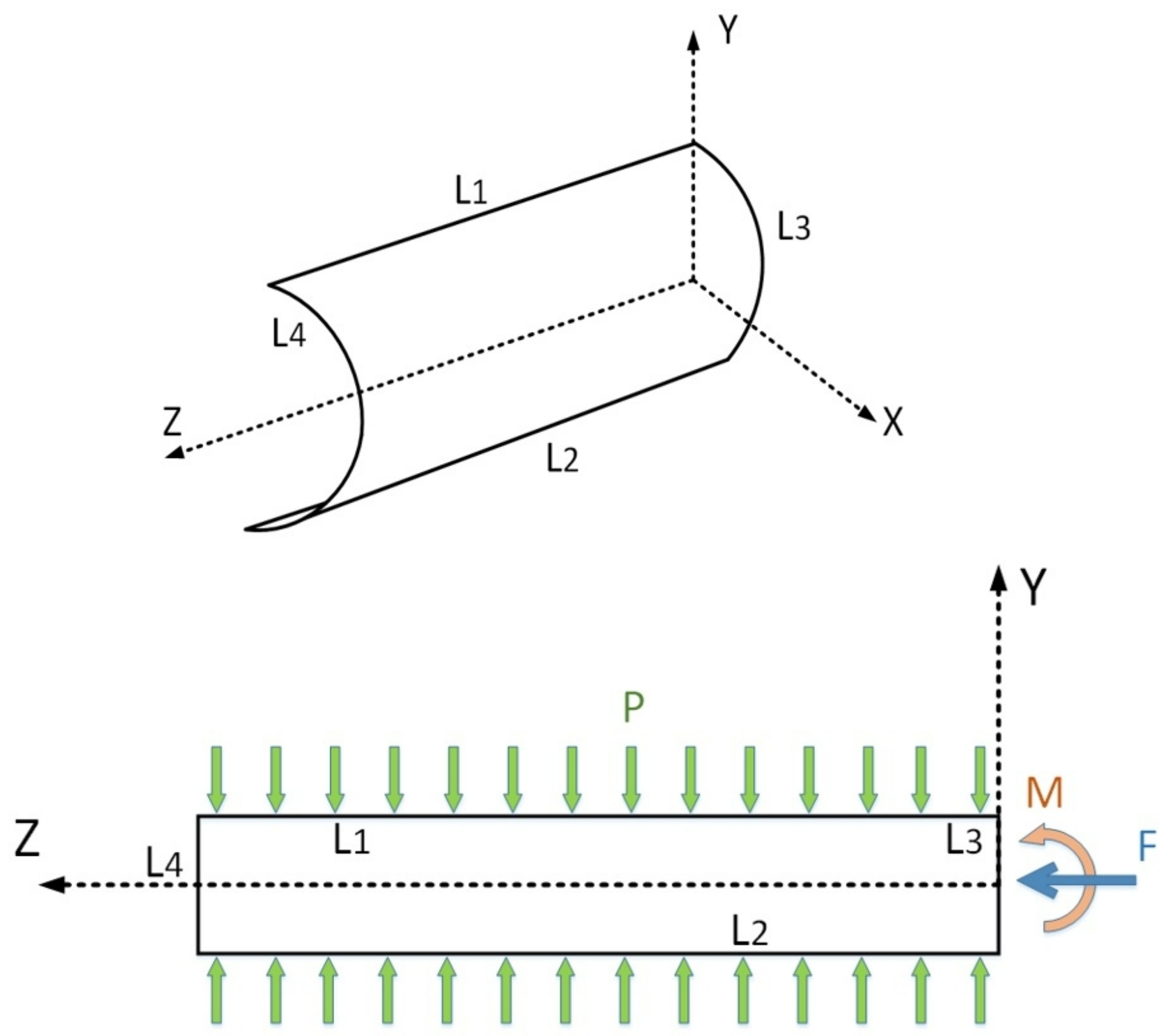


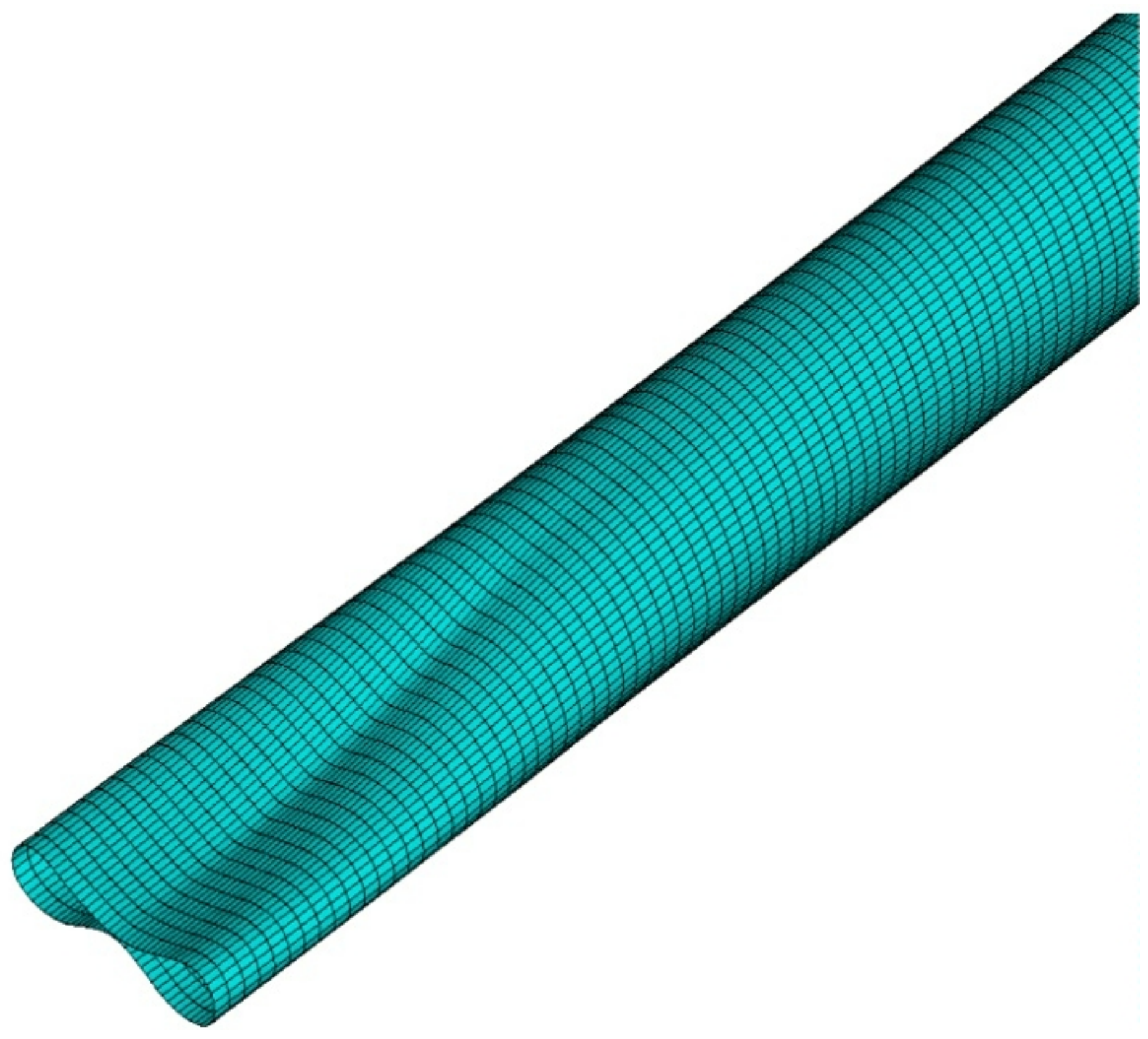


$\frac{M}{M_{P}}$

$L / O D=8.76$

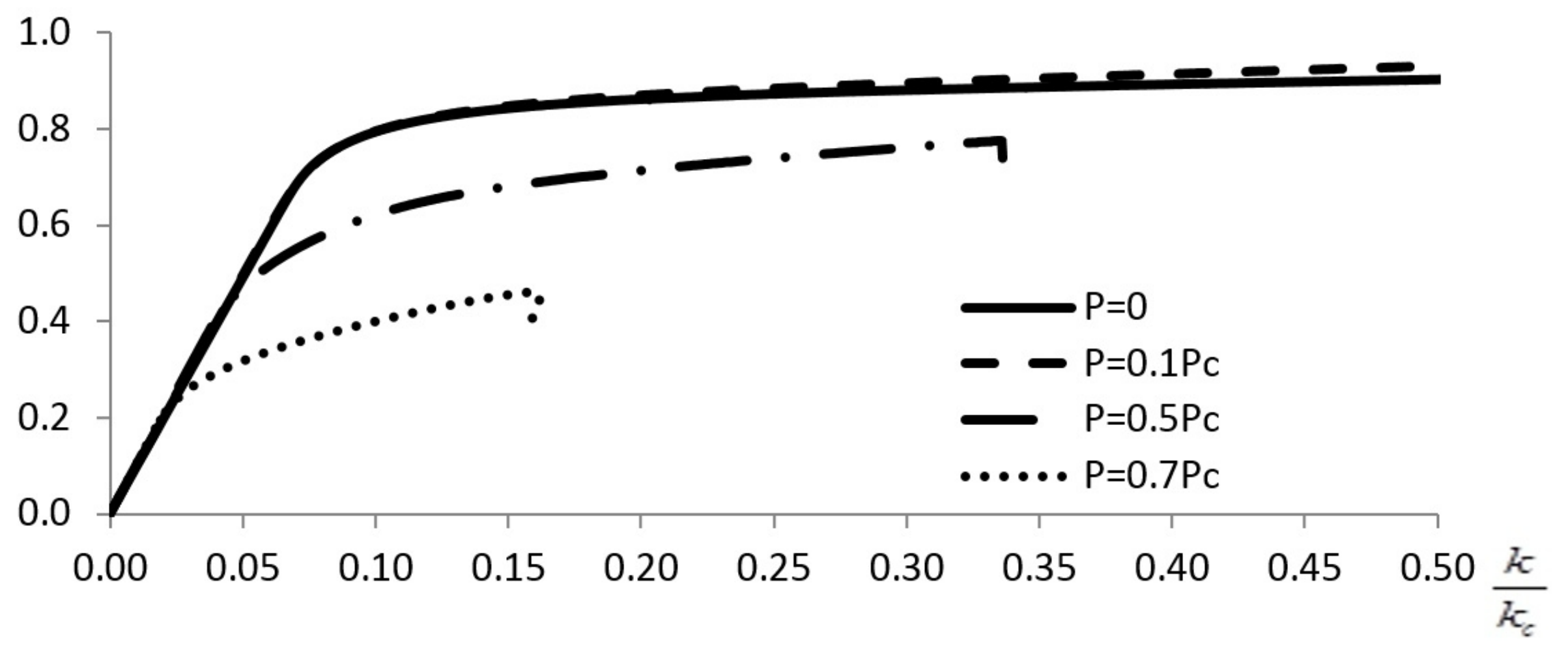




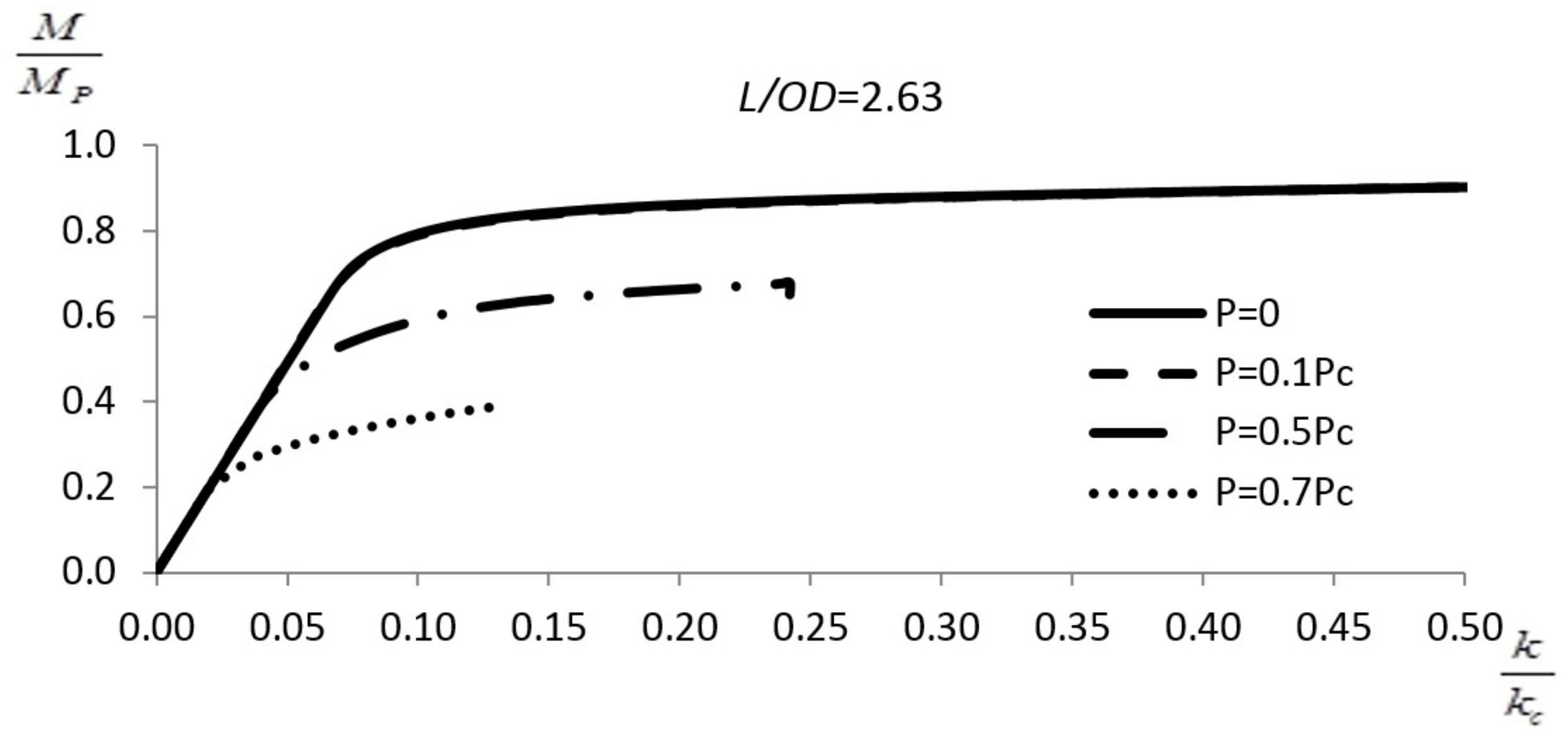


P/Pc

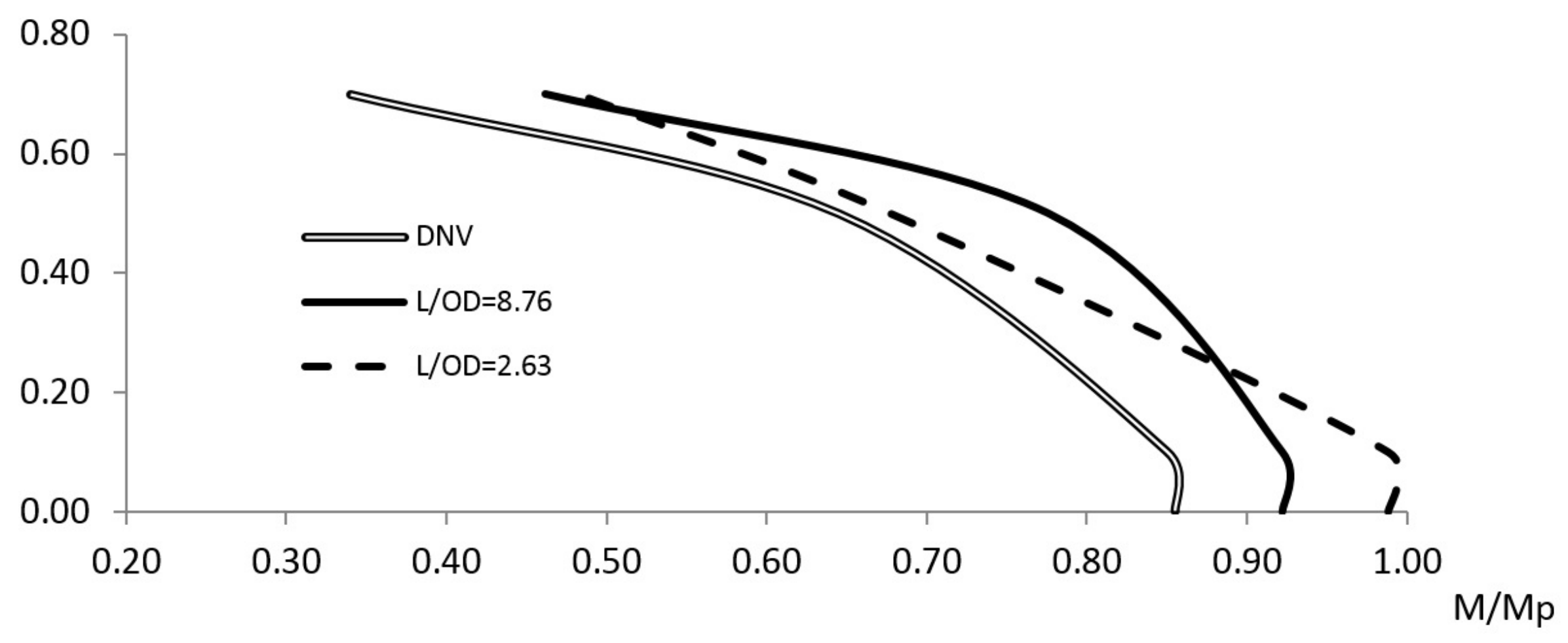


Table 1. Pipe parameters

\begin{tabular}{cccccccccc}
\hline Pipe & $\begin{array}{c}\mathrm{OD} \\
(\mathrm{mm})\end{array}$ & $\begin{array}{c}\mathrm{t} \\
(\mathrm{mm})\end{array}$ & $\begin{array}{c}\mathrm{E} \\
(\mathrm{GPa})\end{array}$ & $v$ & $\begin{array}{c}\mathrm{E}_{\mathrm{t}} \\
(\mathrm{MPa})\end{array}$ & $\begin{array}{c}\sigma_{\mathrm{y}} \\
(\mathrm{MPa})\end{array}$ & $\begin{array}{c}\mathrm{F}_{\mathrm{y}} \\
(\mathrm{kN})\end{array}$ & $\begin{array}{c}\mathrm{M}_{\mathrm{p}} \\
(\mathrm{kN} . \mathrm{m})\end{array}$ & $\begin{array}{c}\mathrm{P}_{\mathrm{c}} \\
(\mathrm{MPa})\end{array}$ \\
\hline 1 & 228.3 & 14.3 & 207 & 0.3 & 2,000 & 450 & 4,326 & 295 & 77.1 \\
2 & 31.70 & 0.91 & 186 & 0.3 & 2,000 & 259 & 11.57 & 223 & 9.67 \\
\hline
\end{tabular}


Table 2. Nonlinear pipe-soil interaction parameters of the constitutive model shown in Fig. 5

\begin{tabular}{|c|c|c|c|c|c|c|c|c|c|}
\hline \multicolumn{6}{|c|}{ Lateral soil resistance parameters } & \multicolumn{4}{|c|}{ Axial soil resistance parameters } \\
\hline $\begin{array}{c}\mathrm{Q}_{0} \\
(\mathrm{~N} / \mathrm{m})\end{array}$ & $\frac{w_{0}}{O D}$ & $\begin{array}{c}\mathrm{Q}_{1} \\
(\mathrm{~N} / \mathrm{m})\end{array}$ & $\frac{w_{1}}{O D}$ & $\begin{array}{c}\mathrm{Q}_{2} \\
(\mathrm{~N} / \mathrm{m})\end{array}$ & $\frac{w_{2}}{O D}$ & $\begin{array}{c}\mathrm{S}_{0} \\
(\mathrm{~N} / \mathrm{m})\end{array}$ & $\frac{r_{0}}{O D}$ & $\begin{array}{c}\mathrm{S}_{1} \\
(\mathrm{~N} / \mathrm{m})\end{array}$ & $\frac{r_{1}}{O D}$ \\
\hline 530 & 0.25 & 740 & 0.50 & 420 & 1.0 & 650 & 0.1 & 280 & 0.25 \\
\hline
\end{tabular}




\section{List of Figures}

Fig. 1. Hobbs lateral buckling mode shapes [4]

Fig. 2. (a) Pipeline with single imperfection and, (b) the nonlinear pipe-soil model adopted in the analytical solution and used for validating the FE model.

Fig. 3. Comparison between analytical and FE lateral buckling forces of pipe-1 represented in Table 1 with as-laid geometry and soil-pipe interaction shown in Fig. 2.

Fig. 4. As-laid configuration of the half-length of the pipeline with two imperfections

Fig. 5. Lateral and axial soil resistance models used in the lateral buckling imperfection study

Fig. 6. Normalised buckle forces plotted against normalised imperfection spacing for (a) $L_{1}=L_{2}=$ $200 O D$ and (b) $L_{1}=L_{2}=100 O D$.

Fig. 7. Normalised buckle load plotted against normalised lateral buckle amplitude for pipeline with single curvature and normal soil with; (a) $L_{I}=L_{2}=200 O D$, (b) $L_{I}=L_{2}=200 O D$, (c) $L_{I}=L_{2}=100 O D$, and (d) $L_{1}=L_{2}=100 O D$.

Fig. 8. Normalised crown moment plotted against normalised buckle amplitude for pipeline on normal soil with $L_{I}=L_{2}=100 O D$ and;

(a) $\Delta_{1}=3.5 \mathrm{OD}, \Delta_{2}=3.5 \mathrm{OD}$,

(b) $\Delta_{1}=3.5 \mathrm{OD}, \Delta_{2}=-3.5 \mathrm{OD}$, $\Delta_{1}=7.0 \mathrm{OD}, \Delta_{2}=3.5 \mathrm{OD}$ and (d) $\Delta_{1}=7.0 \mathrm{OD}, \Delta_{2}=-3.5 \mathrm{OD}$

Fig. 9. Lateral buckle configuration of pipeline in normal soil with and $L_{l}=L_{2}=100 O D$ and $\mathrm{L}_{\mathrm{G}}=20 O D$ with same and opposite sign imperfections.

Fig. 10. Moment vs. lateral buckling curvature response of pipeline with $L_{1}=L_{2}=100 O D$, $\Delta_{1}=7.0 \mathrm{OD}, \Delta_{2}=3.5 \mathrm{OD}$ on a soft soil.

Fig. 11. (a) Exaggerated view of the assumed wrinkled initial imperfection in the vicinity of midlength, (b) Interaction of bending and external hydrostatic pressure for pipe- 2 in Table 1

Fig. 12. A crown segment of the pipeline under the combined actions of lateral buckling (axial force $F$ and bending moment $M$ ) and external pressure $P$.

Fig. 13. (a) FE model of the interaction between lateral and propagation buckling (loading and constraints), (b) FE result of buckle propagation due to the interaction between lateral buckling and external pressure

Fig. 14. Moment vs. curvature response of pipe- 1 in Table 1 under combined external pressure and bending with, a) $L / O D=8.76$, b) and $L / O D=2.63$

Fig. 15. Combined external pressure and bending envelopes: DNV, pipe with $L / O D=8.76$ and 2.63 


\section{List of Tables}

\section{Table 1. Pipe parameters}

Table 2. Nonlinear pipe-soil interaction parameters of the constitutive model shown in Fig. 5 


\section{Manuscript Details}

\section{Manuscript number}

Title
MAST_2017_373_R1

Effect of proximity of imperfections on buckle interaction in deep subsea pipelines

Research Paper

\section{Article type}

Abstract

Lateral buckling of pipelines with closely spaced imperfections laid on even seabed with nonlinear soil-pipe interaction is investigated using finite element analysis (FEA). An analytical solution for lateral buckling of pipeline with single imperfection is proposed and is used to validate the FEA. Effects of amplitude, half wave-length and spacing of imperfections as well as soil resistance on lateral buckling response are presented. It is found that at imperfection spacing greater than half the imperfection half wave-length, there will be no interaction between the buckle lobes. Using validated FEA, the collapse of the pipe-wall due to the interaction between lateral buckling and external pressure is studied. Buckle interaction envelopes are developed and compared to recommendations of the DNV standard. It is shown that in pipeline with closely spaced imperfections, the lateral curvature at the onset of buckling is as large as the critical collapse curvature under combined bending and external pressure.

\section{Keywords}

Corresponding Author

Corresponding Author's Institution

Order of Authors
Lateral buckling Subsea pipeline Buckle interaction Combined bending and external pressure Cylindrical shell Imperfection

Hassan Karampour

Griffith University

Hassan Karampour 
- Lateral buckling of subsea pipeline with closely spaced imperfections is studied using validated FEA.

- Possible interactions between buckles lobes are investigated and results are compared to lateral buckling response of pipeline with single imperfection.

- Using validated FEA, the collapse of the pipe-wall due to the interaction between lateral buckling and external pressure is studied.

- Buckle interaction envelopes are developed and compared to recommendations of the DNV standard. 